\title{
Nanoplasmonic Sensing using Metal Nanoparticles
}

\section{Erik Martinsson}

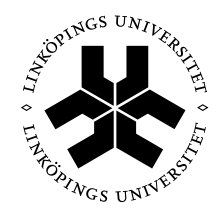

\section{Linköping University \\ INSTITUTE OF TECHNOLOGY}

\author{
Division of Molecular Physics \\ Department of Physics, Chemistry and Biology \\ Linköping University, Sweden
}

Linköping 2014 
Cover: An illustration of surface immobilized spherical gold nanoparticles illuminated with a ray of light.

During the course of the research underlying this thesis, Erik Martinsson was enrolled in Forum Scientium, a multidisciplinary doctoral programme at Linköping University, Sweden.

(C) Copyright 2014 ERIK MARTINSSON, unless otherwise noted

Martinsson, Erik

Nanoplasmonic Sensing using Metal Nanoparticles

ISBN: 978-91-7519-223-9

ISSN: 0345-7524

Linköping Studies in Science and Technology, Dissertation No. 1624

Electronic publication: http://www.ep.liu.se

Printed in Sweden by LiU-Tryck, Linköping 2014. 
"Everything happens for a reason and that reason is usually physics" 



\section{Abstract}

In our modern society, we are surrounded by numerous sensors, constantly feeding us information about our physical environment. From small, wearable sensors that monitor our physiological status to large satellites orbiting around the earth, detecting global changes. Although, the performance of these sensors have been significantly improved during the last decades there is still a demand for faster and more reliable sensing systems with improved sensitivity and selectivity. The rapid progress in nanofabrication techniques has made a profound impact for the development of small, novel sensors that enables miniaturization and integration. A specific area where nanostructures are especially attractive is biochemical sensing, where the exceptional properties of nanomaterials can be utilized in order to detect and analyze biomolecular interactions.

The focus of this thesis is to investigate plasmonic nanoparticles composed of gold or silver and optimize their performance as signal transducers in optical biosensors. Metal nanoparticles exhibit unique optical properties due to excitation of localized surface plasmons, which makes them highly sensitive probes for detecting small, local changes in their surrounding environment, for instance the binding of a biomolecule to the nanoparticle surface. This is the basic principle behind nanoplasmonic sensing based on refractometric detection, a sensing scheme that offers real-time and label-free detection of molecular interactions.

This thesis shows that the sensitivity for detecting local refractive index changes is highly dependent on the geometry of the metal nanoparticles, their interaction with neighboring particles and their chemical composition and functionalization. An increased knowledge about how these parameters affects the sensitivity is essential when developing nanoplasmonic sensing devices with high performance based on metal nanoparticles. 


\section{Populärvetenskaplig sammanfattning}

I dagens samhälle finns en påtaglig vilja och strävan efter att kunna mäta, övervaka, analysera och reglera vardagliga funktioner och beteenden. Detta har lett till en explosionsartad utveckling av nya, funktionella sensorer som numera finns överallt i våra liv - i hemmet, på arbetsplatsen, i våra fickor och till och med inuti våra kroppar. En biosensor är en särskild typ av sensor som används för att detektera specifika kemiska eller biologiska ämnen genom att omvandla förekomsten av dessa ämnen till en mätbar signal. Biosensorer kan användas inom flera olika områden som livsmedelindustri, processövervakning, kriminalteknologi samt inom medicinsk diagnostik. Ofta är dock koncentrationen av de eftersökta ämnena mycket låg vilket ställer stora krav på sensorernas känslighet och precision.

I denna avhandling beskrivs hur nanopartiklar av guld och silver kan användas som signalomvandlare i optiska biosensorer. Metalliska nanopartiklar har unika optiska egenskaper vilket gör dem väldigt känsliga mot små förändringar i dess absoluta närhet. Sådana förändringar ger upphov till en färgförändring hos partiklarna vilket gör det möjligt att optiskt detektera interaktionen med intressanta biomolekyler och därmed utveckla extremt känsliga biosensorer. Nanopartiklarnas minimala storlek underlättar vid integrering i befintliga instrument och möjliggör även skapandet av små, portabla sensoriska system.

Resultaten från detta arbete visar att känsligheten för att detektera förändringar i partiklarnas direkta omgivning kan förbättras avsevärt genom att modifiera nanopartiklarnas storlek, form och kemiska sammansättning. En ökad känslighet medför att lägre koncentrationer av de eftersökta ämnena kan bestämmas, något som är väldigt viktigt för utvecklandet av framtidens bioanalytiska sensorer. 


\section{List of publications}

This thesis is based on the following papers, which are referred to in the text by their Roman numerals (I-IV).

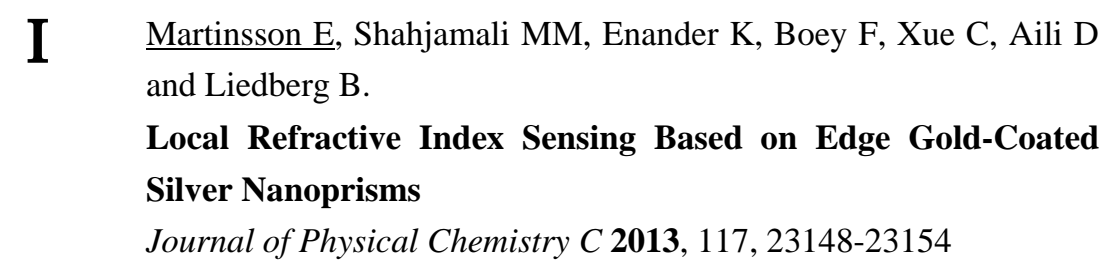

\section{Author's contribution:}

EM was responsible for planning, performing and evaluating the refractive index sensitivity measurements. EM wrote the main part of the paper.

MMS synthesized the nanoparticles.

I Martinsson E, Sepulveda B, Chen P, Elfwing A, Liedberg B and Aili D.

Optimizing the Refractive Index Sensitivity of Plasmonically Coupled Gold Nanoparticles

Plasmonics 2014, 9, 773-780

\section{Author's contribution:}

EM was responsible for planning, performing and evaluating all of the experimental work. EM wrote the main part of the manuscript. BS performed the theoretical calculations. 
D Martinsson E, Otte MA, Shahjamali MM, Sepulveda B and Aili D. Substrate Effect on the Refractive Index Sensitivity of Silver Nanoparticles

Journal of Physical Chemistry C 2014, In Press

\section{Author's contribution:}

EM was responsible for planning, performing and evaluating the refractive index sensitivity measurements. EM wrote the main part of the manuscript. MMS synthesized the nanoparticles. MAO and BS performed the theoretical calculations.

IV Martinsson E, Shahjamali MM, Large N, Zaraee N, Schatz GC, Mirkin CA and Aili D.

Influence of Surfactant Bilayers and Substrate Immobilization on the Refractive Index Sensitivity of Anisotropic Gold Nanoparticles

In manuscript

\section{Author's contribution:}

EM was responsible for planning, performing and evaluating the refractive index sensitivity measurements. MMS synthesized the nanoparticles. EM and MMS wrote the main part of the manuscript. NL performed the theoretical calculations. 


\title{
Papers not included in the thesis
}

\author{
V Jönsson C, Aronsson M, Rundström G, Pettersson C, Mendel- \\ Hartvig I, Bakker J, Martinsson E, Liedberg B, MacCraith B, \\ Öhman O and Melin J. \\ Silane-Dextran Chemistry on Lateral Flow Polymer Chips for \\ Immunoassays
}

Lab on a Chip 2008, 8, 1191-1197

VI Shahjamali MM, Bosman M, Cao S, Huang X, Saadat S, Martinsson E, Aili D, Tay YY, Liedberg B, Chye S, Loo J, Zhang H, Boey F and Xue C.

\section{Gold Coating of Silver Nanoprisms}

Advanced Functional Materials 2012, 22, 849-854

VI Shahjamali MM, Martinsson E, Marcello W, Yin L, Liedberg B, Boey F and Xue C.

Edge Gold-Coated Silver Nanoprisms [Ag@(Au Nanoframe)] for $\mathrm{H}_{2} \mathrm{O}_{2}$ Detection

Asia Pacific Optical Sensors Conference 2012, 83511S-5 


\section{Abbreviations}

\begin{tabular}{|c|c|}
\hline AFM & Atomic Force Microscopy \\
\hline APTES & (3-Aminopropyl) Triethoxysilane \\
\hline \multirow[t]{2}{*}{ BSPP } & bis( $p$-Sulfonatophenyl) Phenylphosphine Dihydrate \\
\hline & Dipotassium \\
\hline $\mathrm{CO}$ & Carbon Monoxide \\
\hline CTAC & Cetyltrimethylammonium Chloride \\
\hline CTAB & Cetyltrimethylammonium Bromide \\
\hline DDA & Discrete Dipole Approximation \\
\hline FDTD & Finite-Difference Time-Domain \\
\hline FOM & Figure of Merit \\
\hline FWHM & Full-Width-Half-Maximum \\
\hline IR & Infrared \\
\hline LBL & Layer-By-Layer \\
\hline LSPR & Localized Surface Plasmon Resonance \\
\hline MPTES & (3-Mercaptopropyl) Triethoxysilane \\
\hline NSL & Nanosphere Lithography \\
\hline PAH & Polyallylamine Hydrochloride \\
\hline PEF & Plasmon-Enhanced Fluorescence \\
\hline PEG & Polyethylene Glycol \\
\hline PSS & Polystyrene Sulfonate \\
\hline QCM & Quartz Crystal Microbalance \\
\hline RI & Refractive Index \\
\hline RIU & Refractive Index Unit \\
\hline SAM & Self-Assembled Monolayer \\
\hline SEM & Scanning Electron Microscopy \\
\hline SERS & Surface-Enhanced Raman Spectroscopy \\
\hline SP & Surface Plasmon \\
\hline SPR & Surface Plasmon Resonance \\
\hline TEM & Transmission Electron Microscopy \\
\hline
\end{tabular}




\section{Contents}

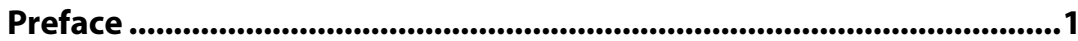

1. Introduction ...........................................................................................................3

Nanoplasmonic sensing............................................................................. 5

\section{Synthesis and fabrication of metal nanoparticles}

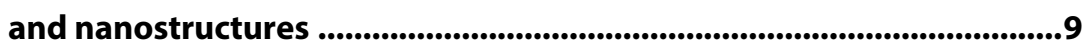

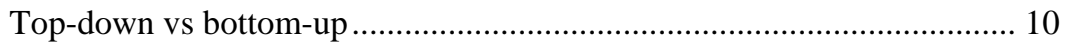

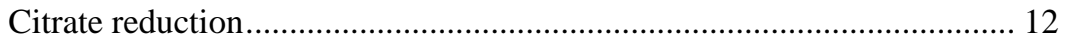

Shape-controlled seed-mediated growth ..................................................... 13

Photoinduced conversion synthesis.......................................................... 15

3. Optical properties of metal nanoparticles ..................................................17

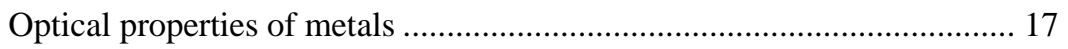

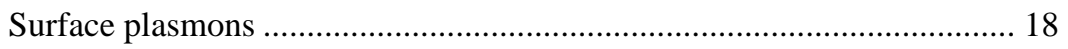

Localized surface plasmons .................................................................... 20

Optical response of anisotropic nanoparticles - beyond the Mie theory ... 23

Plasmonic coupling .................................................................................. 24

4. Functionalization and immobilization of metal nanoparticles.............27

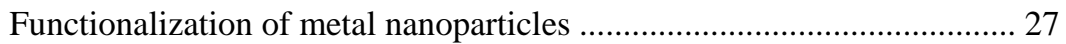

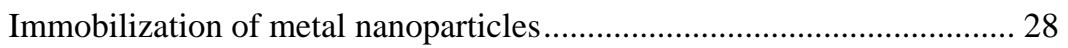

Controlling the surface coverage of metal nanoparticles ........................... 29

Nanoparticle assembly on silanized surfaces ............................................ 31

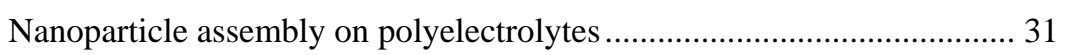

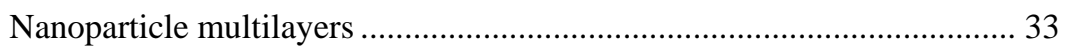

5. Plasmonic biosensing...........................................................................................35

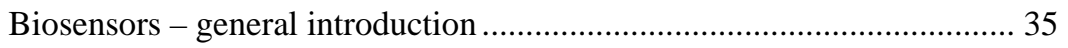

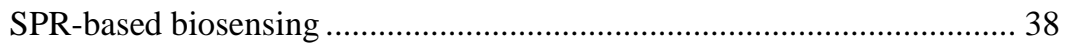

LSPR-based refractometric biosensing .................................................... 41

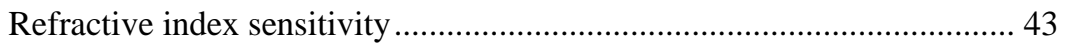

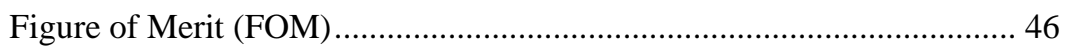

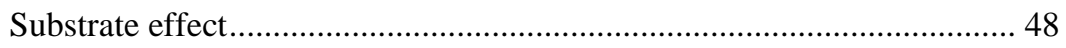




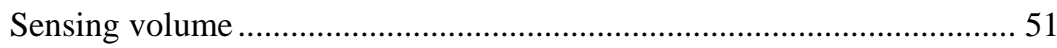

6. Summary of papers...........................................................................................55

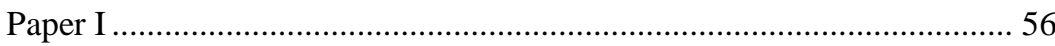

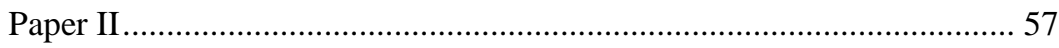

Paper III .............................................................................. 58

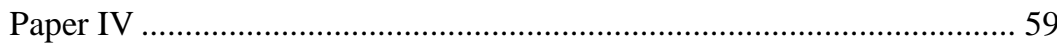

7. Future perspective...............................................................................61

References ................................................................................................65 


\section{Preface}

Nanoplasmonics explores the formation and application of surface plasmons in metal nanostructures. This topic, which belongs to the field of nanophotonics, enables a way to confine and control light at a nanoscale and promise new, exciting applications within a broad range of technological areas ranging from molecular diagnostics to quantum computers. Nanoplasmonics is indeed a fascinating and intriguing scientific field and it has been a pleasure to enter and explore this world during my years as a Ph.D. student. I am convinced that we only have seen the beginning of this emerging topic and that the future will include a great number of technical inventions and products based on nanoplasmonics.

This thesis focuses on nanoplasmonic sensing and is divided into seven chapters. The first chapter provides an introduction to the world of plasmonics and nanoplasmonic sensing. The synthesis and fabrication of plasmonic nanomaterials is covered in chapter 2 and the optical properties of these materials are described in chapter 3 . Chapter 4 focuses on functionalization and surface immobilization of metal nanoparticles. Optical biosensing, which is the central topic of this thesis, is covered in chapter 5. A short summary of the 
included papers are presented in chapter 6 . Finally, chapter 7 is devoted to future perspective and challenges for nanoplasmonic sensing.

The main work presented in this thesis has been conducted at the Department of Physics, Chemistry, and Biology (IFM) at Linköping University within the group of Molecular Physics. The work that has resulted in this thesis could not been achieved without the help and encouragement from numerous people. I would like express my sincere gratitude and appreciation to some particular people who have helped me throughout my years at IFM.

First, I would like to acknowledge my main supervisors Bo Liedberg for accepting me as a Ph.D. student and giving me the freedom to pursue my own ideas and Daniel Aili for the excellent guidance and enthusiasm during the last hectic time. Thanks also to Karin Enander and Thomas Ederth for chairing some of their great wisdom with me.

Many thanks to my closest collaborators and co-authors of the papers, especially Mohammad Shahjamali for providing me with all the magnificent nanoparticles. Thanks also to Borja Sepulveda, Marinus Otte and Nicolas Large for their invaluable assistance with the theoretical calculations.

All past and present members in the group of Molecular Physics are also acknowledged for fruitful discussions in the lab and at the group meetings.

Many thanks to all my wonderful friends within the orienteering community for helping me to "relax" during my spare time.

Finally, but most importantly, I would like to thank my family especially Sara for unconditional support and for always believing in me no matter what.

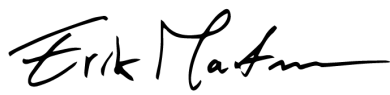

Linköping, October 2014 


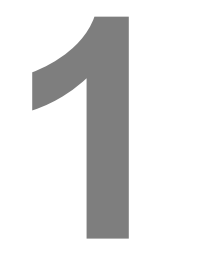

\section{Introduction}

When matter is reduced into nanoscale structures, new unique physical properties emerge that are not seen in bulk material. This has fascinated mankind for centuries and forms the basis of nanotechnology a scientific and technological field that has grown tremendously during the last decades. Nanotechnology deals with materials, systems, and devices on the nanometer length scale ( 1 - 100 nanometer) and the concept was first described in 1959 by Richard Feynman in his famous lecture entitled "there's plenty of room at the bottom". ${ }^{1}$ In his talk he accurately predicted the direction of modern nanotechnology and nanoscience, playing with the idea of miniaturization and atomic engineering. Today, many of the things he anticipated have become reality and we now possess the ability to create and manipulate material at nanoscale dimensions. This has made a significant impact on modern society and the number of applications and products that include nanomaterials increases steadily. Nowadays, nanotechnology is well-established within the manufacturing industry and a respectable scientific field that involves numerous researchers from a wide range of areas including physics, chemistry, biology, medicine, electronics, and engineering. 
Nanoplasmonics is a field within nanotechnology that utilizes the unique physical and optical properties of metals., ${ }^{2,3}$ These properties are strongly associated with a phenomena known as localized surface plasmon resonance (LSPR), ${ }^{4-6}$ which arises when free electrons in metal nanostructures are excited, creating collective electron oscillations confined in the nanostructures (more information about plasmons in chapter 3). Excitation of localized surface plasmons can be induced by electromagnetic radiation (light) which results in strong scattering and absorption at specific wavelengths which gives metallic nanomaterial distinctive colours. The vibrant colours of nanoplasmonic materials have captivated humans for a long time and have been used to decorate glass, ceramics, and mosaics for centuries. One of the most famous artifacts utilizing the optical properties of noble metal nanostructures is the Lycurgus cup (Figure 1.1), which is a Roman glass cup dated from the fourth century, currently exhibited at the British Museum in London. The cup is of dichroic nature, exhibiting a green jade colour in ambient light and a deep ruby red colour when illuminated from inside. Several analyses have been conducted on the cup in order to understand its optical features. ${ }^{7-9}$ These studies have revealed that the
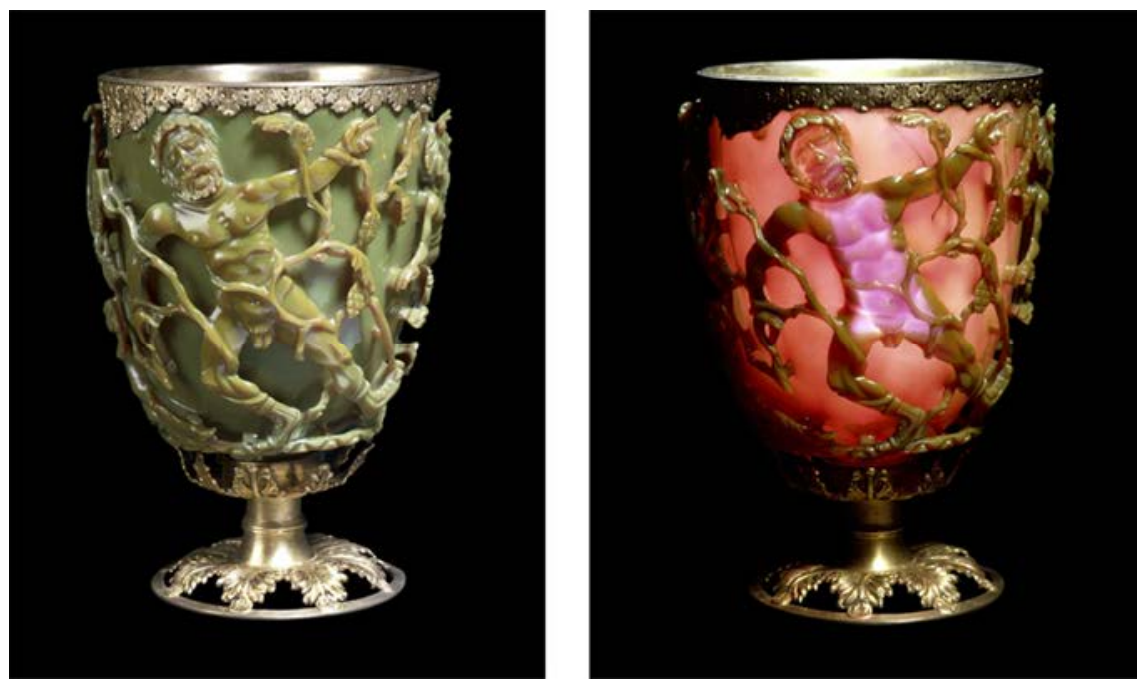

Figure 1.1: The Lycurgus cup illuminated with ambient lighting (left) and illuminated from inside (right) (from the British Museum free image service). 
cup contains gold-silver (30:70) alloy nanoparticles, 50-100 nm in diameter, embedded in the glass and which absorbs and scatters light at around $515 \mathrm{~nm}$. Although, there are several other examples of glass stained with metal nanoparticles from this era, the Roman glassmakers most certainly produced these objects without knowing that it was actually metal nanoparticles that gave rise to these wonderful colours. Today, nanoplasmonic materials are not primarily used to stain glass anymore but they have, however, found several other applications in many different areas such as optical waveguides, ${ }^{6,10-12}$ photovoltaics, ${ }^{13-15}$ catalysis $^{16,17}$ and finally within chemical and biological sensing. ${ }^{5,18-26}$

\section{Nanoplasmonic sensing}

The excitation of localized surface plasmons in metallic nanostructures using visible light makes nanoplasmonic materials especially attractive for optical sensing applications. When incident light interacts with metal nanostructures, the photons are either absorbed or scattered which is greatly enhanced at the resonance frequency which can be monitored using optical spectroscopy based either on extinction or scattering measurements. When this interaction occurs and light is confined and the energy is converted into a localized surface plasmon, a strong electromagnetic field is created in the direct vicinity of the nanostructures. This highly localized field induced by the LSPR excitation makes metal nanostructures sensitive probes for detecting small, local variations in the surrounding environment. ${ }^{5,18,20}$ A local change in the refractive index (RI), e.g. due to adsorption of proteins or other biomolecules, can be detected since spectral changes occurs in the light used for LSPR excitation. This forms the basis of nanoplasmonic sensing a simple yet sensitive strategy for detecting biological or chemical interactions. Nanoplasmonic sensing offers real-time, label-free molecular detection and shows a great potential for miniaturization and multiplexing due to the small dimensions of the metal nanoparticles. Refractometric sensing using plasmonic nanostructures have for example been used to detect biomarkers for Alzheimer's disease ${ }^{27,28}$ as well as for cancer. ${ }^{29}$ However, most clinically relevant biomarkers are present at very low concentrations, which mean that the sensitivity is a very important aspect to consider when developing bioanalytical sensing devices, in particular for 
diagnostics. Nanoplasmonic sensors are also employed for a wide number of non-medical applications, such as environmental pollution control, food testing and detection of chemical warfare agents and explosives. In all of these sensing applications, the sensitivity is of critical importance for the overall performance of the sensor system. Methods to improve the sensitivity in LSPR-based sensors have therefore been an area of intense research.

In nanoplasmonic refractometric sensing, the sensitivity for detecting small, local changes in the RI close to the metal nanostructures is commonly defined and measured as changes in either the plasmon peak position or intensity per refractive index unit (RIU). These measurements are normally performed by monitoring the spectral changes that occurs when plasmonic materials are exposed to solutions with a known RI. The RI sensitivity is highly dependent on the nature of the plasmonic nanoparticles i.e. their size, shape, and metal composition but also on the interaction with other metal nanoparticles. ${ }^{30,31}$ Thus, there are several parameters that influence the sensitivity and by varying the structure, morphology, and surroundings of the plasmonic nanomaterial it is possible to tune the sensitivity and hence, improve the performance of a nanoplasmonic sensing system based on RI detection.

The aim of the work presented in this thesis has been to explore the RI sensitivity of metal nanoparticles made by gold and/or silver, two commonly used plasmonic materials. Both the bulk and surface RI sensitivity of several different types of nanoparticles has been examined as well as how interparticle coupling and surface immobilization affects the sensitivity. A comprehensive overview on factors influencing the RI sensitivity and possibilities to optimize the RI sensitivity is presented, providing valuable information for the production of sensing devices utilizing plasmonic nanoparticles as transducer elements.

This thesis is based on four papers, which all focus on the RI sensitivity of metal nanoparticles. In Paper I, we studied both the bulk and local RI sensitivity of edge-gold coated silver nanoprisms and compared them to both conventional surface plasmon resonance (SPR) and spherical gold nanoparticles. Paper II focuses on how the RI sensitivity of gold nanospheres can be increased by utilizing plasmonic coupling between nanoparticles separated by polyelectrolytes layers. In Paper III, we examined both experimentally and 
theoretically, how a surface immobilization to a solid support (glass) affects the sensitivity of three types of silver nanoparticles of different shapes. This is an important aspect to consider since most nanoplasmonic sensing devices are based on immobilized nanoparticles rather than suspended particles. Finally, the influence of a common stabilizing agent (CTAX) on the RI sensitivity is explored in Paper IV, where a simple method based on oxygen plasma treatment is employed in order to increase the RI sensitivity of nanoparticles immobilized on a solid substrate. 


\title{
2
}

\section{Synthesis and fabrication of metal nanoparticles and nanostructures}

\begin{abstract}
Although, metal nanomaterial have been used for a long time it was not until the mid- $19^{\text {th }}$ century that the properties of metal nanoparticles was first examined and described by Michael Faraday. ${ }^{32}$ He was fascinated by the colours of metal nanoparticles and was able to produce an aqueous solution of gold particles by reducing gold salt with phosphorus. He suspected that the colour of the suspension was caused by the presence of very fine particles of gold that were "very minute in their dimensions" and he also concluded that "a mere variation in the size of its particles gave rise to a variety of resultant colours" but he could not confirm their presence or explain the colour alterations. Some decades later, Richard Zsigmondy continued the work with colloidal nanoparticles and together with Henry Siedentopf he developed the ultramicroscope where single gold nanoparticles could be observed and their nanosize dimensions could be verified. $^{33}$ After the pioneering work of Faraday and Zsigmondy, colloidal chemistry and the production of nanomaterials have advanced considerably and during the past decades, a variety of different methods have been developed, to
\end{abstract}


create well-defined nanostructures with various properties. ${ }^{34-36}$ The advancements in nanofabrication techniques have enabled production of nanoparticles and nanopatterns with great control over their size, shape, and separation. Combined with an increasing knowledge in surface chemistry and functionalization, metal nanostructures have become versatile and functional building blocks for a large variety of applications.

Although the progress in this area has been substantial in the recent years there is still need for improvements, in particular with respect to the production of highly-defined metal nanostructures using large-scale, high-throughput fabrications techniques. The sensitivity and performance of nanoplasmonic sensing devices can be significantly improved if nanoparticles with high uniformity and stability can be synthesized since a narrow size distribution will result in a sharper LSPR band, which means that smaller spectral shifts can be detected. Also, since any structural modification of metal nanoparticles will affect their RI sensitivity, it is crucial that the nanoparticles exhibit a high morphological stability and remains unaltered after functionalization and when exposed to a complex media. Thus, further development in the synthesis and fabrication of metal nanomaterials are necessary in order to develop commercially viable and highly sensitive nanoplasmonic sensing platforms.

\section{Top-down vs bottom-up}

The construction of nanomaterials can be divided into two major production techniques - top-down fabrication and bottom-up assembly. ${ }^{37}$ Top-down fabrication techniques utilize different lithographic methods in order to produce patterned nanostructures whereas bottom-up methods are based on the assembly of atoms, molecules, and other components into nanostructures either on substrates or in solution. Top-down lithography methods, such as electron beam lithography, can produce plasmonic materials with great control over the size, shape, and spacing of metal nanostructures. ${ }^{38}$ This enables a production of highly reproducible plasmonic substrates where the optical properties can be tuned, which allows for systematic investigations of various plasmonic applications such as Surface-Enhanced Raman Spectroscopy (SERS), ${ }^{39-41}$ Plasmon-Enhanced Fluorescence (PEF), ${ }^{42,43}$ and refractometric sensing. ${ }^{44,45}$ However, a major 
disadvantage of conventional lithography techniques is the problem to realize large areas with nanopatterns at a reasonable cost. Because of this reason, Van Duyne and his group developed a technique called nanosphere lithography (NSL), which is an inexpensive nanofabrication method that enables production of larger nanopatterned areas. ${ }^{46,47}$ NSL utilize micron sized beads, made for instance by silica or polystyrene, that are immobilized on a substrate and act as a mask during metal deposition. When removing the beads, a nanopattern is developed from the metal that was deposited in the voids between the beads. NSL can produce well-ordered arrays of nanostructures with uniform size and shape in a large format $\left(>1 \mathrm{~cm}^{2}\right)$ at relativity low cost. ${ }^{48}$ There are, however, apparent limitations to the structural features that can be fabricated using this technique because of the geometry of the beads used as mask.

NSL is considered to be a hybrid method that utilizes both top-down fabrication and bottom-up assembly and is good example on how these two nanofabrication techniques have converged during the last decades (Figure 2.1). Today, both techniques can be used to create structures with nanometer precision in essentially the same size regime.

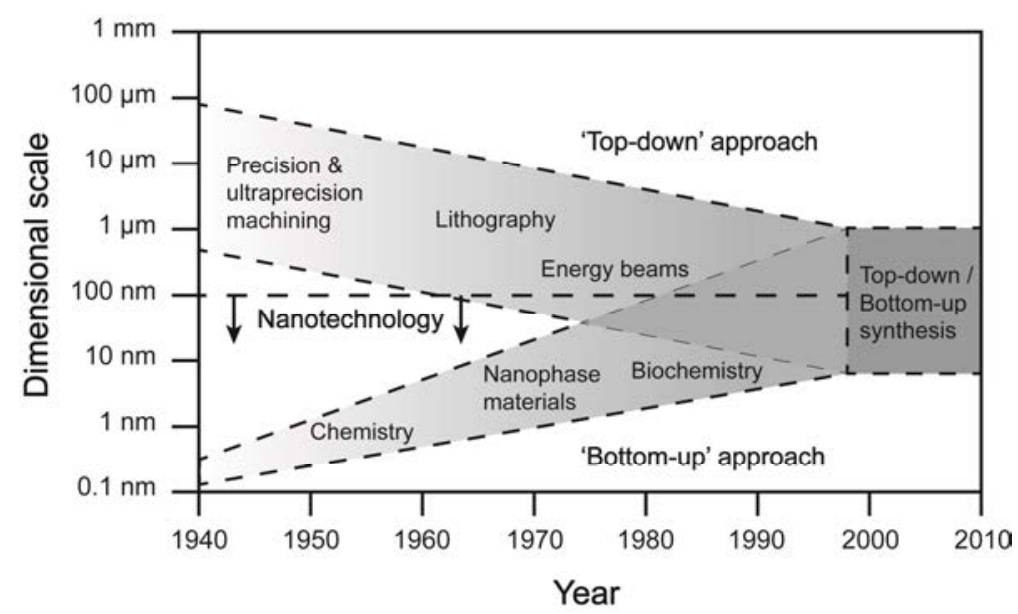

Figure 2.1: The convergence of top-down and bottom-up approaches. Adopted from ${ }^{49}$. 
Compared to top-down lithographic techniques the bottom-up assembly approach is generally a faster and cheaper way to produce desirable nanomaterials for various applications. The bottom-up approach provides a huge flexibility and a large variety of structures can be created. The power of selfassembly becomes very evident when looking at the structural and compositional complexity of natural materials and nanostructures. The challenge is thus, to control the forces and physical boundary conditions involved in the assembly when molecules and nanostructures form larger aggregates, in order to produce functional and well-defined materials. This in fact also applies to synthesis of plasmonic nanoparticles, where elaborate procedures have been developed to control the arrangement of metal atoms to realize nanoparticles of various sizes, shapes, and compositions. ${ }^{35,50,51}$

The work included in this thesis is based solely on metal nanoparticles fabricated by bottom-up solution phase synthesis where the particles either have been kept in suspension or immobilized on a solid support using self-assembly.

\section{Citrate reduction}

A common strategy to synthesize metal nanoparticles is to dissolve and reduce metal salt in the presence of molecules that can prevent the nanoparticles from aggregating. The concentration ratio of reduction agent to metal salt affects the growth kinetics of the nanoparticles and thus affects their size. Often the reduction agent also stabilizes the nanoparticles thus filling a dual role. One example is the method first described by Turkevich et al. in $1951,{ }^{52}$ where gold chloride $\left(\mathrm{HAuCl}_{4}\right)$ was reduced by sodium citrate generating fairly monodispersed gold nanoparticles suspended in an aqueous solution. This method was later improved by Frens who produced gold particles with a diameter of $\sim 10-150 \mathrm{~nm} .{ }^{53}$ In this procedure, citrate acts both as a reducing agent and electrostatic stabilizer where the size of the particles can be controlled by varying the ratio between the gold salt and the citrate (Figure 2.2). The reaction temperature is also important for the formation of the metal nanoparticles since both the reaction kinetics and oxidation potential are dependent upon the temperature. The excess of citrate anions forms a complex multilayer around the particles which prevent aggregation and gives the particles a net negative surface 
charge. ${ }^{50}$ The moderately weak coordination of citrate makes the particles amenable for surface functionalization with for example proteins or other biomolecules. However, the citrate capping makes the particles sensitive to changes in $\mathrm{pH}$, ionic strength in the medium, and the presence of other organic materials which complicates the functionalization procedure significantly. ${ }^{34,54}$
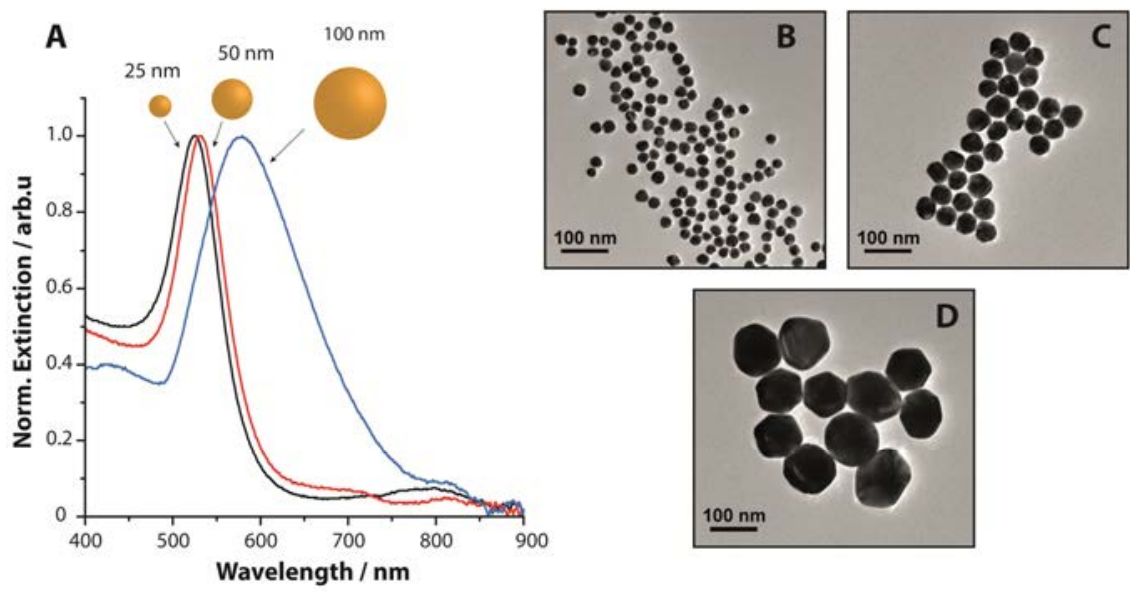

Figure 2.2: (A) Extinction spectra and (B-D) transmission electron microscopy (TEM) images of gold nanoparticles of different sizes produced by citrate reduction.

\section{Shape-controlled seed-mediated growth}

Generally, the growth mechanism for metal nanoparticles can be divided into two steps, a nucleation followed by a controllable growth of the existing nuclei. If only metal salt and a reducing agent are added to the reaction, spherical nanoparticles will be formed since they possess the lowest surface energy. ${ }^{55}$ In order to produce anisotropic nanoparticles the growth reaction needs to be directed to specific crystal facets which promote growth in a particular direction. A popular technique for shape-controlled synthesis of metal nanoparticles is the seed-mediated growth method. ${ }^{26,34,50,56-58}$ In this method, tiny seeds are first created that act as nucleation sites. In the second step, a mild reducing agent (e.g. ascorbic acid) is used to facilitate further growth without initiating further 
nucleation, i.e. formation of new seeds. By adding various capping agents to the initial seeds that preferentially bind to specific crystal facets, the growth rate is reduced at these facets, guiding the growth of the nanoparticle into certain geometries. The size and shape of the particles are controlled by varying the type and concentration of the reagents used in the reaction as well as the reaction conditions. One of the most common capping agents used in order to control the shape of gold nanoparticles is an ionic surfactant called cetyltrimethylammonium (CTAX) where $\mathrm{X}=\mathrm{Cl}^{-}$or $\mathrm{Br}^{-}$(Figure 2.3). CTAX has been extensively employed for the synthesis of various gold nanoparticles with different geometries and thus different optical properties, including nanorods, ${ }^{58}$ nanocubes, ${ }^{59}$ nanostars, ${ }^{60}$ nanoprism ${ }^{61}$ etc. However, a major disadvantage with CTAX as a capping agent is that it binds very strongly to the metal nanoparticles, making it particularly hard to chemically replace the CTAX with other more biocompatible and biofunctional molecules. Moreover, CTAX is known to be highly cytotoxic. ${ }^{62-64}$ Various strategies have, however been examined in order to exchange the CTAX with different ligands to increase the biocompatibility and reduce the cytotoxicity. ${ }^{62-65}$ For instance, Niidome et al. replaced the CTAX on gold nanorods with thiol-PEG (Polyethylene glycol), which resulted in a significantly lower cytotoxicity, thus enabling the usage of gold nanorods for in vivo applications. ${ }^{62}$ Gold nanoparticles stabilized with CTAX has been synthesized and were exploited in Paper IV 

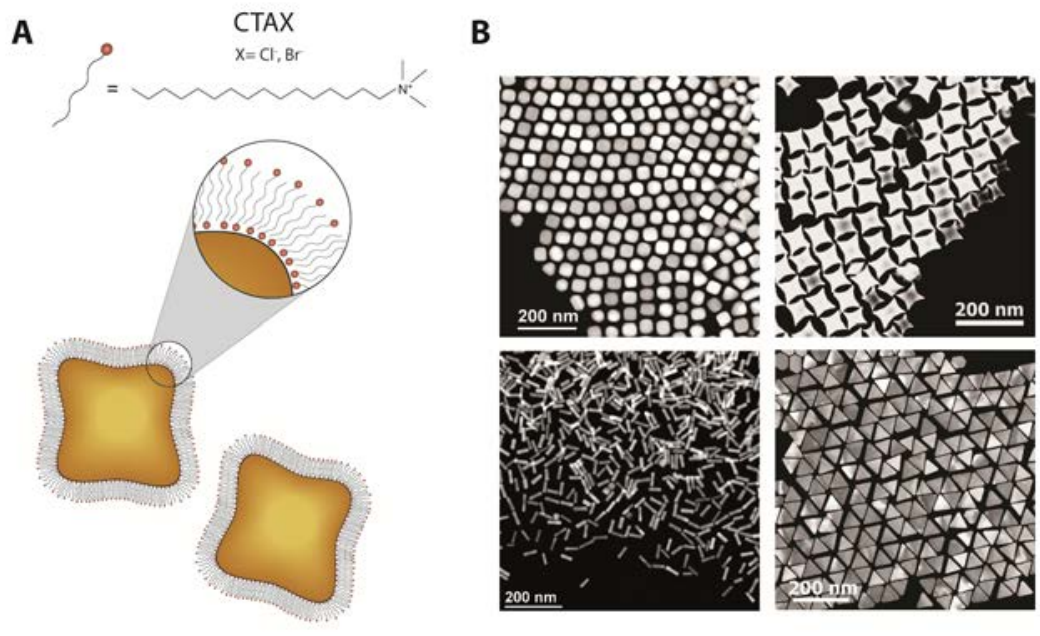

Figure 2.3: (A) Illustration of CTAX-capped nanoparticles and (B) TEM images of four differently-shaped gold nanoparticles produced with CTAX.

\section{Photoinduced conversion synthesis}

Anisotropic nanoparticles are particularly attractive for optical biosensing due to the presence of enhanced electromagnetic near-fields, especially around sharp edges and tips. ${ }^{66,67}$ Due to this reason, triangular nanoparticles or nanoprisms have gained a great deal of interest from the sensing community. A versatile synthesis route for producing triangular silver nanoparticles in high yield was reported by Mirkin et al. in 2001. ${ }^{68}$ They showed that silver nanospheres could be transformed into triangular nanoprisms using a photoinduced conversion procedure. By irradiating a solution containing small silver nanospheres $(\sim 8 \mathrm{~nm}$ in diameter) with visible light in the presence of sodium citrate and $\operatorname{bis}(p$ sulfonatophenyl) phenylphosphine dihydrate dipotassium salt (BSPP), triangular nanoprisms were formed with an edge length of about $100 \mathrm{~nm}$. Later, it was shown that the size of the nanoprisms could be controlled by varying the wavelength of the irradiation light. ${ }^{69}$ Consequently, since the optical properties are highly dependent on the structure and morphology of the nanoparticles, colloids with tunable LSPR bands and colors ranging from blue to red could be prepared. 
Unfortunately, a major drawback with silver nanoparticles is that they can easily undergo oxidation, leading to structural changes which significantly affect their optical properties. Nanostructures with sharp-tip features like nanoprisms are especially sensitive since an oxidation leads to a truncation of the tips resulting in nanodiscs or nanoplates with reduced electromagnetic near-fields as compared to the prisms. In order to preserve the shape of the nanoprisms and retain their desirable optical properties a thin layer of gold can be deposited on the edges of the silver nanoprisms. ${ }^{70} \mathrm{~A}$ schematic illustration of a synthetic route for producing edge-gold coated silver nanoprisms can be seen in Figure 2.4. By coating the silver nanoprisms with gold their morphology and optical features are maintained and they exhibit a better stability against oxidation. The optical properties and RI sensitivity of gold-coated silver nanoprisms were examined in

\section{Paper I.}
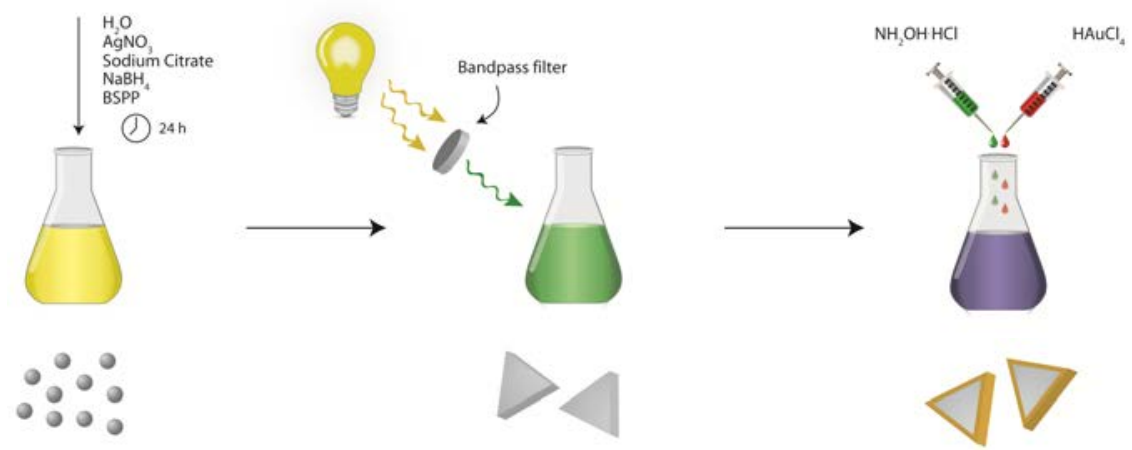

Figure 2.4: Schematic illustration of a synthesis route for making gold-coated silver nanoprisms. 


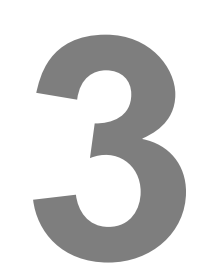

\section{Optical properties of metal nanoparticles}

\section{Optical properties of metals}

The optical properties of bulk materials can be characterized by the dielectric function $\varepsilon(\omega)$ which describes how materials interact with electromagnetic radiation. For metals, which have partially filled electron bands, the dielectric function can be described with a simple, free-electron model.

$$
\varepsilon(\omega)=1-\frac{\omega_{p}^{2}}{\omega^{2}+i \Gamma \omega}
$$

This is known as the Drude model which assumes that metals consists of a free electron gas also known as plasma that moves within a lattice of fixed, positively charged ion cores. 
If the electron damping factor, $\Gamma$ is neglected, the dielectric function can be further simplified to

$$
\varepsilon(\omega)=1-\frac{\omega_{p}^{2}}{\omega^{2}}
$$

where $\omega_{p}$ is the plasma oscillation frequency of the free electron gas relative to the positively charged ion cores. The plasma oscillation can be calculated using the equation

$$
\omega_{p}=\sqrt{\frac{n_{e} e^{2}}{m_{e} \varepsilon_{0}}}
$$

where $n_{e}$ is the density of electrons, $e$ is the electric charge, $m_{e}$ the effective electron mass, and $\varepsilon_{0}$ is the permittivity of vacuum.

A quantum of plasma oscillation is known as a plasmon, or plasmon polariton when induced by photon excitation. Plasmons can be created in metal bulk material, at metal surfaces or in metal nanostructures which all possess different optical properties.

\section{Surface plasmons}

Plasmons are longitudinal collective electron density fluctuations that when formed at a metal surface, create a surface-bound electromagnetic wave, known as a surface plasmon (SP). ${ }^{71,72}$ This SP wave propagates at the interface between the metal and a dielectric, which is schematically illustrated in Figure 3.1 A. The propagating SP wave has an accompanying electric field that decays exponentially both in the direction of propagation, due to energy losses to the metal, and perpendicular to the interface into both the metal and the dielectric medium. The decay length depends on the dielectric properties of the metal and the surrounding media. 
The energy for an SP wave is always smaller than that of bulk plasmons and the plasmon frequency is $\omega_{S P}=\omega_{p} / \sqrt{2}$.

A propagating SP wave carries momentum and by solving Maxwell's equations with specific boundary conditions, an expression for the dispersion relation of an SP can be derived

$$
k_{S P}=\frac{\omega}{c} \sqrt{\frac{\varepsilon_{d} \varepsilon_{m}}{\varepsilon_{d}+\varepsilon_{m}}}
$$

where $\mathrm{k}_{\mathrm{SP}}$ is the wave vector for the SP and $\varepsilon_{m}$ and $\varepsilon_{d}$ is the dielectric function of the metal and the dielectric surrounding, respectively.

For an SP to be excited both the frequency (energy) and the wave vector (momentum) need to conserved, which cannot be achieved by simply illuminating a metal surface with light passing through air. Figure 3.1 B shows the dispersion relation for an SP compared to photons in vacuum and since the curves do not coincide, the excitation requirements cannot be fulfilled. However, in 1968 Kretschmann and Raether showed that SPs can be excited in flat metal films using ordinary light by guiding the incident light through a coupling medium e.g. a quartz prism. ${ }^{73}$ If the metal film is thin enough the photons can penetrate through the metal film and excite an SP wave on the opposite side of the film at the metal-dielectric interface, where the excitation of SPs is dependent on the thickness of the metal film. ${ }^{72}$ SPs can however be generated at a thick metal film by introducing a spacer layer between the metal and the prism where the RI of the spacer material needs to be lower than that of the prism. The electromagnetic field from the incident light can couple with the metal through the spacer and excite an SP at the metal interface. This is known as the Otto configuration. ${ }^{74}$ Grating coupling is another technique developed in order to excite SPs using electromagnetic waves, where a roughened metal surface is used instead of a smooth surface which allows for direct excitation without coupling through a prism. ${ }^{75}$ 
A

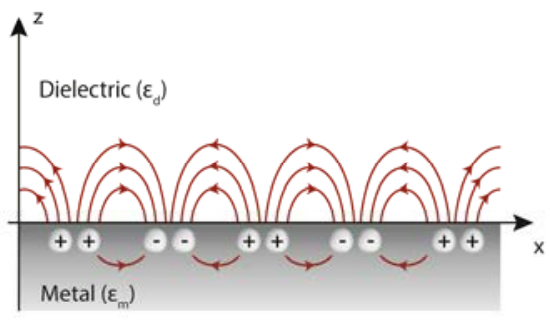

B

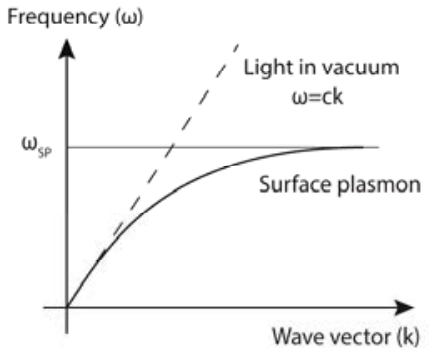

Figure 3.1: (A) Schematic illustration of a surface plasmon propagating along the $x$-axis on the interface between a metal and dielectric with electric field lines in the opposite direction. (B) Dispersion relation for a surface plasmon and for photons in vacuum.

If the RI of the dielectric medium $\left(\varepsilon_{d}\right)$ at the metal interface is altered, the resonance condition for exciting the SP changes, as seen from Equation 3.4. This is the basic principle behind SPR-based biosensing which is further explored in chapter 5 .

\section{Localized surface plasmons}

Discrete metal nanostructures cannot support propagating SPs since there is simply no room for the electrons to propagate. Instead, free electrons in metal nanostructures can undergo excitation and create a collective oscillation restricted by the geometrical boundaries of the nanostructure. These oscillations are known as localized surface plasmons and can be excited by an external electromagnetic field. Since localized SPs do not carry any momentum, only the energy needs to be matched in order to excite the electrons which mean that free photons can be used for excitation and no momentum matching is needed. Consequently, exploration of localized SPs can be done by rather simple optical equipment.

The interaction between light and small metal nanoparticles was first described by Gustav Mie over a century ago. ${ }^{76} \mathrm{He}$ analytically solved Maxwell's equations and derived an expression for metal nanoparticles in a homogenous medium interacting with an electromagnetic field. For spherical metal nanoparticles much 
smaller than the wavelength of light $(2 r<<\lambda)$ the optical extinction cross section $\left(\sigma_{\text {ext }}\right)$ can be expressed with the following relationship:, 5

$$
\sigma_{e x t}(\lambda)=\frac{24 \pi^{2} r^{3} \varepsilon_{d}^{3 / 2} N}{\lambda \ln (10)} \frac{\varepsilon_{i}(\lambda)}{\left(\varepsilon_{r}(\lambda)+2 \varepsilon_{d}\right)^{2}+\varepsilon_{i}(\lambda)^{2}}
$$

where $r$ is the radius of the nanoparticle, $\varepsilon_{d}$ is the dielectric constant of the surrounding medium, $N$ is the electron density, and $\varepsilon_{r}$ and $\varepsilon_{i}$ are the real and imaginary part of the complex dielectric function of the bulk metal, respectively. In this quasi-static approximation, the small nanoparticles are assumed to experience a uniform static electric field and they can thereby be considered as electrical dipoles. The polarizability ( $\alpha$ ) for such a dipole can be expressed as:

$$
\alpha(\lambda)=4 \pi r^{3} \frac{\varepsilon(\lambda)-\varepsilon_{d}(\lambda)}{\varepsilon(\lambda)+2 \varepsilon_{d}(\lambda)}
$$

As seen from Equation 3.6, maximum polarizability occurs when the term in the denominator approaches zero, hence when $\varepsilon(\lambda) \approx-2 \varepsilon_{d}(\lambda)$. The dielectric permittivity of the surrounding medium $\left(\varepsilon_{d}\right)$ can normally be considered as a constant and real parameter, which means that the real part in the dielectric function of the metal nanoparticle $\left(\varepsilon_{r}\right)$ is required to possess a negative value in order for strong polarization to occur, if the imaginary part of the dielectric function is small (low losses). From Equation 3.5, it is clear that maximum extinction arises when this condition is fulfilled, and plasmon resonance occurs. Introducing the Drude dielectric function gives the resonance frequency for a dipolar localized surface plasmon. ${ }^{77}$

$$
\omega_{L S P}=\frac{\omega_{p}}{\sqrt{1+2 \varepsilon_{d}}}
$$

For silver and gold nanoparticles, this frequency falls in the visible region of the spectrum. Consequently, these nanoparticles exhibit bright colours both in transmitted and reflected light (see chapter 1). 
When plasmon resonance occurs, the conduction electrons are displaced relative to the nuclei, moving them away from their equilibrium position. The coulombic attraction between the relocated electrons and the atomic cores acts as a restoring force on the free electrons, which gives rise to an oscillating electron motion at a specific frequency. This oscillation typically decays within a few femtoseconds, ${ }^{78}$ but when an alternating electric field is introduced a continuous coherent plasmon oscillation can be maintained (Figure 3.2). The plasmon excitation gives rise to a strongly enhanced electric field in close vicinity of the nanoparticles, which is utilized in several field-enhanced plasmonic applications. ${ }^{71,77}$

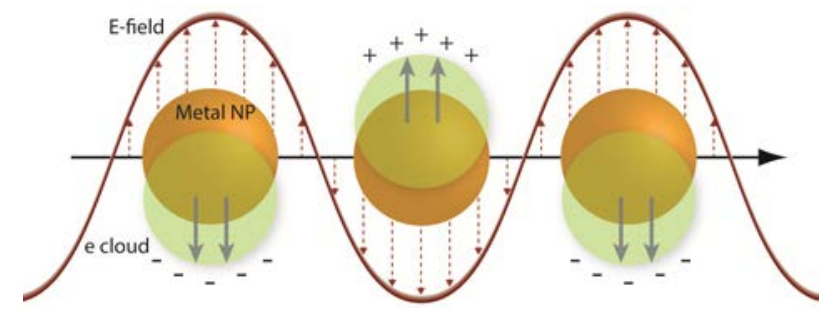

Figure 3.2: Schematic illustration of localized surface plasmon resonance induced by an external electrical field.

When the dielectric medium changes close to the nanoparticles e.g. by biomolecular adsorption to the metal surface, the condition for resonance is altered. An increase in the dielectric function of the environment, gives rise to an increased screening of the surface charges, which weakens the restoring force on the electrons. As a result, less energy is required to excite the electrons which red-shifts (longer wavelength) the plasmon resonance frequency. This is also obvious from Equation 3.7, where an increase in $\varepsilon_{d}$ results in a lower plasmon frequency hence, a longer resonance wavelength. This makes metal nanoparticles sensitive probes for detecting local RI changes, where the sensitivity depends on the polarizability and the strength of the restoring force, which is a function of the material and the geometry of the nanoparticles. ${ }^{77}$ Refractometric sensing based on metal nanoparticles is covered in chapter 5 . 


\section{Optical response of anisotropic nanoparticles - beyond the Mie theory}

For small spherical nanoparticles, the Mie theory can be considered as a simple but efficient model to describe the optical response. However, when the particle size increases the quasi-static approximation is no longer valid since the electrons do not experience a homogenous electric field. This causes a dephasing of the conduction electrons and a retardation of the dipolar field. ${ }^{79}$ The retardation effect lowers the excitation energy which causes a red-shift of the plasmon resonance wavelength for larger nanoparticles. Additionally, radiative losses increase when the particle size gets larger which broadens the plasmon band width and reduces the intensity.

Another way to tune the plasmon frequency is to change the geometry of the nanoparticles. A modification of the nanoparticle shape has a significant impact on the spectral position of the plasmon resonance. Spherical nanoparticles with high symmetry only possess one dipolar resonance, but when the shape is modified and the particles become more asymmetric, multiple dipolar modes can arise which makes the optical response more complex. ${ }^{80}$ For instance, elongated metal nanoparticles like nanorods display both a transverse and a longitudinal localized surface plasmon mode which gives rise to two distinct plasmon resonance peaks at different spectral positions. Several other geometrical features in addition to elongation, affects the actual plasmon frequency such as curvature, asymmetry and intracoupling. ${ }^{71}$ Furthermore, higher-order plasmon modes can be created due to an inhomogeneous distribution of the surface charges in the nanoparticles. Such multimodes (e.g. quadropoles, octopoles) are normally created in larger nanoparticles and are always located at shorter wavelengths (higher energy) compared to the dipolar excitation. ${ }^{81-83}$ Consequently, by varying the size and/or shape of the nanoparticles, plasmon peaks over the entire visible and near-infrared region can be generated.

The optical response from anisotropic nanoparticles of different shape, size, and material can be calculated with the help of various numerical methods. Two of the most commonly used methods are discrete dipole approximation (DDA) and finite-difference time-domain (FDTD) ${ }^{84}$ DDA calculations are based on a threedimensional array of small, polarizable elements considered as point dipoles. The dipole moment induced by an external electric field and through interaction 
with other dipoles, is calculated for each element. The contribution from each dipole is then used to calculate the overall optical response, i.e. the scattering and absorption of the incident light caused by the metal nanoparticles. ${ }^{85,86}$ FDTD simulations are based on solving Maxwell's differential equations in a threedimensional cubic lattice over time. The optical properties of the material are defined in each cell and the electric and magnetic fields are calculated at a given instant of time. FDTD enables simulation of the electromagnetic response from heterogeneous materials of arbitrary geometries. ${ }^{87-89}$ Computational simulation techniques are important in order to increase the knowledge of the physics behind plasmonics and to verify and explain experimentally obtained results.

\section{Plasmonic coupling}

So far, only single nanoparticles have been considered. However, electromagnetic interactions between adjacent nanoparticles give rise to an additional spectral shift of the plasmon resonance position due to Coulomb interactions. This is known as plasmonic coupling and results in new hybridized plasmon modes. ${ }^{90}$ The interaction can either form a "bonding" plasmon mode (lower energy) or an "anti-bonding" plasmon mode (higher energy) depending on the polarization of the surface charges. The simplest model is to consider two closely spaced spherical metal nanoparticles separated with a distance (d). As seen in Figure 3.3 A, the spectral position of the plasmonic coupling mode is highly dependent on the interparticle separation and red-shifts significantly when the distance between the particles is reduced. Utilization of plasmonic coupling is hence another powerful way to tune the plasmon resonance frequency, which is employed in Paper II. ${ }^{31,91}$

Plasmonic coupling between closely spaced metal nanostructures generates large near-field enhancement in the gap between the particles, usually called "hot spots”. The induced local electrical field can be enhanced by a factor of x100 compared to the surrounding electric field. ${ }^{92}$ Figure 3.3 shows the formation of a hot spot in a gold nanoparticle dimer separated by $3 \mathrm{~nm}$ (B) and $10 \mathrm{~nm}$ (C). Clearly, a significantly higher field strength occurs when the particles are closely spaced, separated by only $3 \mathrm{~nm}$. These hot spots are especially interesting for SERS, where the large field strengths can be used to increase the Raman 
scattering signals from specific molecules placed inside the enhanced nearfields. $^{93,94}$
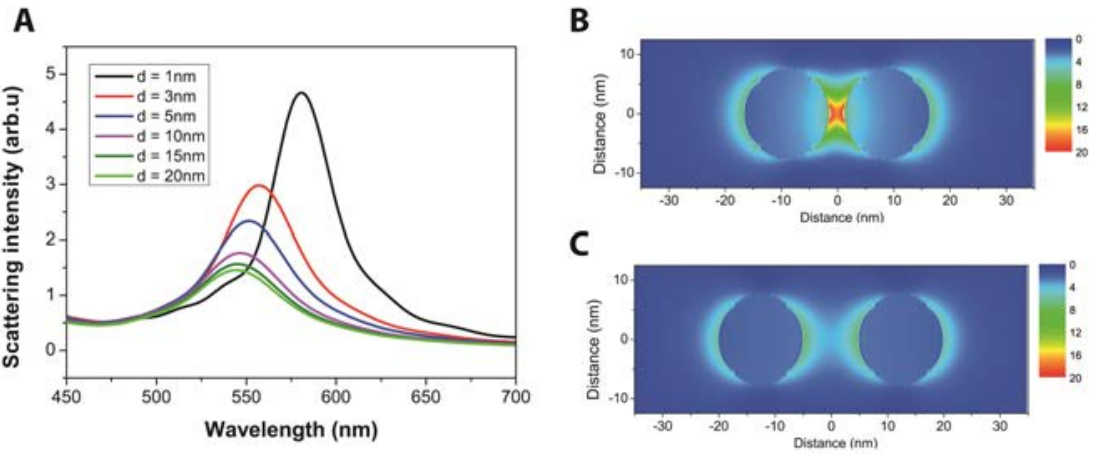

Figure 3.3: The plasmonic coupling between individual nanoparticles is significantly dependent on the interparticle separation (d). (A) Scattering spectra acquired with FDTD calculations of two gold nanoparticles (10 $\mathrm{nm}$ in diameter) at different particle separations. Corresponding field plots for (B) $\mathrm{d}=3 \mathrm{~nm}$ and (C) $\mathrm{d}=10 \mathrm{~nm}$. Courtesy of Dr. Borja Sepulveda (CIN2, Barcelona)

The large resonance shift created when metal nanoparticles are brought in close proximity is used in a biosensing method called colorimetric sensing. This method was pioneered by Mirkin et al. who used DNA to facilitate an aggregation of gold nanoparticles. ${ }^{95}$ Several other sensing strategies, based on metal nanoparticle aggregation have later been developed, detecting a large variety of molecular species. $^{96,97}$ 


\section{4}

\section{Functionalization and}

\section{immobilization of metal}

nanoparticles

\section{Functionalization of metal nanoparticles}

In order to convert metal nanoparticles to useful, hybrid nanomaterials, they typically need to be modified with some organic material that provides them with a desired functionality. ${ }^{98}$ A chemical modification of the metal surface is normally required in order to introduce specificity and to increase the biological compatibility of the metal nanoparticles in sensing applications. Functionalization of metal nanoparticles generally requires a modification or replacement of the stabilizing agent that is used in the synthesis to prevent the particles from aggregating. Thus, the new surface chemistry must maintain particle stability and at the same time provide the particles with new chemical properties and functionalities. Biomolecules can be conjugated to nanoparticles through physisorption (noncovalent coupling) or by chemisorption (covalent coupling). ${ }^{98}$ This can be done directly on the particle surface or with the 
assistance of a bifunctional crosslinker. Regardless of the choice of conjugation method, it is a great challenge to control the amount of material bound to the surface as well as the orientation of the biomolecules in order to achieve an accessible surface chemistry with high specificity for binding of analytes.

Extensive work has been conducted on metal nanoparticle functionalization which has resulted in a huge amount of protocols for introducing different chemical moieties using both covalent and non-covalent approaches. The most frequently employed method to functionalize gold nanoparticles is indisputably the usage of thiol chemistry. Thiol groups (-SH) form covalent bonds with gold atoms, forming a self-assembled monolayer (SAM) on gold surfaces. ${ }^{99}$ As thiols are present in, or can be introduced into several different classes of molecules (e.g. DNA, polypeptides etc.), gold nanoparticles can easily be functionalized with a large variety of biomolecules. Physisorption of, for instance, polymers or proteins is another commonly applied method to functionalize nanoparticles. ${ }^{98}$ In addition, they can also be coated with inorganic materials like silica in order to make them more chemically inert (silver nanoparticles) and suspendable in various solvents. ${ }^{100,101}$

\section{Immobilization of metal nanoparticles}

Dispersed nanoparticles need to be stabilized in order to prevent them from aggregating. However, if the particles are tethered to a solid support, it is no longer necessary to uphold a chemical or steric repulsion between them since strongly attached nanoparticles are unable to move laterally across the surface and thereby incapable of aggregating. For this reason, substrates with nanoparticles organized into ordered structures like arrays or other controlled assemblies, has found their way into many electronic, optical, and sensor applications. ${ }^{102}$

The optical properties of metal nanoparticles are normally studied using optical spectroscopy. However, an exploration of immobilized nanoparticles generally requires the use of high magnification imaging techniques. Electron microscopy techniques like scanning electron microscopy (SEM) or transmission electron 
microscopy (TEM) are especially useful for studying metal nanoparticles since their high electron densities gives a very high contrast in the images. For this reason, metal nanoparticles can be used as contrast agents in various microscopy techniques. ${ }^{103}$ Atomic force microscopy (AFM) is also routinely used to study particle assembly and morphology on various substrates.

\section{Controlling the surface coverage of metal nanoparticles}

Immobilization of nanoparticles on a solid support usually involves surface modification followed by particle adsorption using either pre-functionalized or non-treated particles, where the particle adhesion is driven by electrostatic forces and van der Waals dispersion forces. Controlling the surface density of particles attached on the substrate is very important in order to achieve an appropriate surface morphology with desired functionalities.

If the particles are exposed to a surface that enables adsorption, the binding kinetics primarily depends on the properties of the particle solution. ${ }^{104}$ In the initial adsorption process, we can assume that every nanoparticle that touches the surface will adhere and stick to it, irreversibly. Then the number density $\left(N_{\text {surface }}\right)$ can be calculated using the following expression: ${ }^{104}$

$$
N_{\text {surface }}=2 c_{0} \sqrt{D_{\text {bulk }} t / \pi}
$$

where $c_{0}$ is the bulk concentration of nanoparticles, $t$ is the incubation time and $D_{\text {bulk }}$ is the diffusion constant given by the well-known Stokes-Einstein equation:

$$
D_{b u l k}=\frac{k_{B} T}{6 \pi \eta R}
$$

where $k_{B}$ is Boltsmann's constant, $T$ is the absolute temperature, $\eta$ is the viscosity of the medium, and $R$ is the radius of a spherical particle. As seen from 
Equation 4.1, the surface density of particles on the surface depends on the particle concentration $\left(c_{0}\right)$ and the incubation time $(t)$ and can hence be considered as a first-order rate process. ${ }^{105}$ The particle concentration and the incubation time can rather easily be varied in order to control the surface coverage of nanoparticles. A high surface coverage with short interparticle distances, gives rise to plasmonic coupling between the closely packed metal nanoparticles. This is normally an unwanted effect since the distinct plasmon resonance band originating from individual nanoparticles can be significantly broadened.

Most metal nanoparticles suspended in aqueous media are stabilized by charged species at their surface, such as citrate, and this charge repulsion will also affect the surface coverage. If the repulsion is decreased (shorter Debye length) the nanoparticles can form a closer packing and hence, a higher surface coverage can be obtained. The electrostatic repulsion between particles can be controlled by varying the ionic strength in the solution. ${ }^{105} \mathrm{~A}$ density gradient of immobilized gold nanoparticles was created by Lundgren et al. by changing the ionic strength in the nanoparticle solution by ion diffusion. ${ }^{106}$

Figure 4.1 shows SEM images of gold nanoparticles immobilized on substrates with different surface coverages. Different surface coverages were obtained because of variations in the particle concentration in the different particle suspensions.
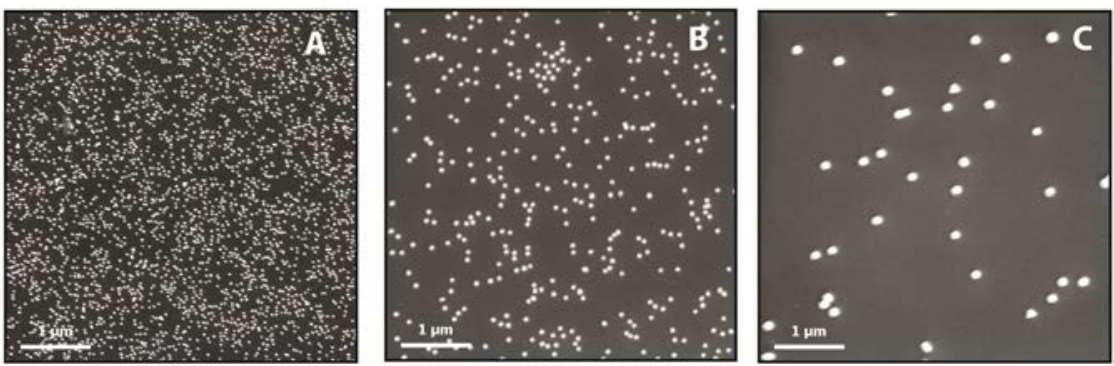

Figure 4.1: SEM images of substrates covered with gold nanoparticles of various sizes with different surface coverage. (A) 12\% (25 nm), (B) $7 \%$ (50 nm) and (C) $3 \%(100 \mathrm{~nm})$. 


\section{Nanoparticle assembly on silanized surfaces}

Silanization is a straightforward method to introduce functional chemical groups onto silica-based substrates like glass. Thus, silanization is a frequently used surface modification in order to immobilize nanoparticles or biomolecules onto glass substrates. ${ }^{105}$ A self-assembled monolayer of silanes is created by the interaction with hydroxyl group on the surface, forming a covalent Si-O-Si bond. ${ }^{107}$ Prior to the silanization process, the surfaces must be thoroughly cleaned in order to remove organic material and increase the amount of hydroxyl groups present on the surface. A well-defined silanized monolayer is crucial for obtaining a successful colloidal immobilization.

Two frequently used organosilanes utilized for nanoparticle attachment is (3aminopropyl) triethoxysilane (APTES) and (3-mercaptopropyl) triethoxysilane (MPTES) (Figure 4.2). In Paper II and III, silanization with APTES was used for surface immobilization of metal nanoparticles on glass substrates.

A

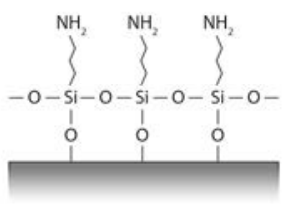

B

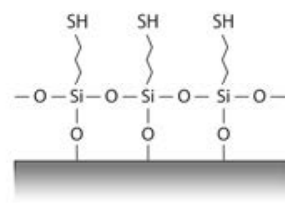

Figure 4.2: Surfaces functionalized with two different silanes, (A) APTES and (B) MPTES.

\section{Nanoparticle assembly on polyelectrolytes}

Polyelectrolyte multilayer formation is a versatile layer-by-layer (LBL) technique which enables a nanoscale arrangement of organic molecules where materials are sequentially adsorbed on top of each other. ${ }^{108}$ LBL methods can be used to sequentially build-up thin films with nanometer-scale precision which has several applications in a large variety of fields, including nanoparticle functionalization and immobilization. Polyelectrolyte multilayers are formed by exploiting the electrostatic attraction between oppositely charged polymer chains. 
The adsorption of molecules with different charge has two important consequences: i) repulsion of equally charged molecules limits the adsorption to the formation of a single monolayer in each sequence and ii) the ability of an oppositely charged molecule to be adsorbed on top of the first one. ${ }^{108}$ This allows for the formation of a multilayer molecular structure if these steps are repeated in a cyclic manner.

The formation of a multilayered structure comprised of oppositely charged polyelectrolytes give rise to a charged surface, where the top polyelectrolyte layer determines the surface charge. This enables immobilization of charged nanoparticles using electrostatic interactions between the surface charges on the nanoparticles and the top layer on the polyelectrolyte coated surface. Since the surface charge can be altered by simply changing the top polyelectrolyte layer, both negatively and positively charged nanoparticles can be immobilized using this method.

Two specific polyelectrolytes have been extensively used in the work included in this thesis, polyallylamine hydrochloride (PAH) which is a cationic polyelectrolyte and polystyrene sulfonate (PSS) which is anionic. These are two of the most frequently used polyelectrolytes for LBL formation and their properties have been comprehensively examined by others. ${ }^{109-112}$ One bilayer of $\mathrm{PAH} / \mathrm{PSS}$ results in a thickness in air of about $3 \mathrm{~nm}$ (Figure 4.3), which was determined using null ellipsometry. However, the thickness of the polymer film significantly depends on the ionic strength in the deposition solution, since an increase of the electrolyte concentration results in a more effective screening of the polyelectrolyte charges, which changes the conformation of the polymers. $^{111,112}$ Also, polyelectrolytes multilayers are known to swell and increase in thickness when exposed to water. A swelling of $\sim 30 \%$ for a polymer film composed of PAH/PSS has been reported by others. ${ }^{113}$ 


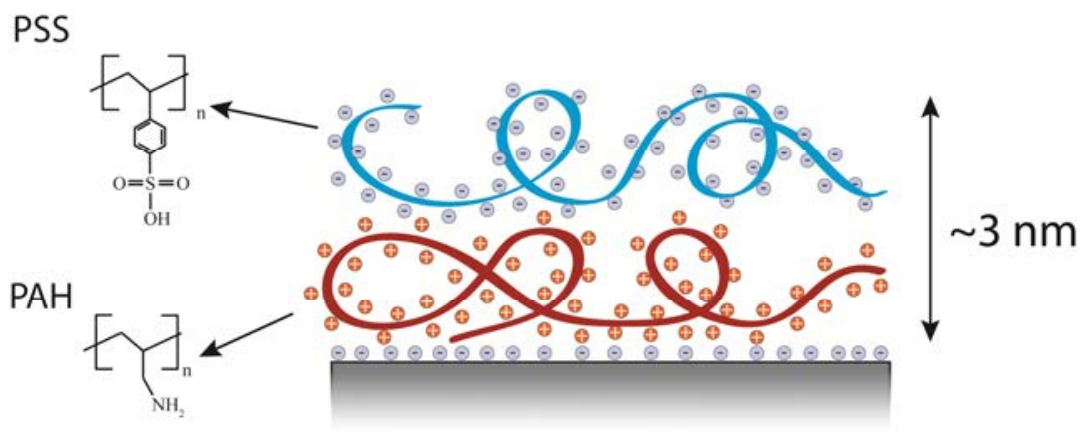

Figure 4.3: A polyelectrolyte bilayer composed of polyallylamine hydrochloride (PAH) and polystyrene sulfonate (PSS). The thickness (3 nm) was obtained using null ellipsometry in air.

As each layer deposition enables a thickness control on the nanometer-scale, a layer-by-layer assembly of polyelectrolytes can also be used for probing the surface sensitivity and sensing depth of a refractometric sensing system, which was exploited in Paper I. Also, polyelectrolyte multilayers can be used to control and fine-tune interparticle distances (Paper II).

\section{Nanoparticle multilayers}

In order to create a multilayer structure of nanoparticles, the initial monolayer must be modified in such a way that adsorption of a second particle layer is possible. This can be achieved by using a variety of different surface chemistry modifications with molecular components such as DNA, ${ }^{114}$ peptides, ${ }^{115}$ or polymers. ${ }^{116,117}$ Widely separated layers of nanoparticles can significantly increase the extinction of light (Figure 4.4), which can be useful for applications where surfaces with high extinction (absorption and scattering) are desired. Hence, 3D-assembly is yet another way to tune the optical response from plasmonic nanoparticles.

If the distance between the layers is reduced, plasmonic coupling can occur which gives rise to new plasmon bands. If the distance between the layers is not 
precisely defined, many plasmon resonance modes will be present which gives a broad and undefined plasmon band. The spacing between each monolayer of particles depends solely on the material used to achieve the multilayer formation. Plasmonic coupling with distinct plasmon bands was achieved in Paper II, using a protocol based on polyelectrolytes as spacer material.

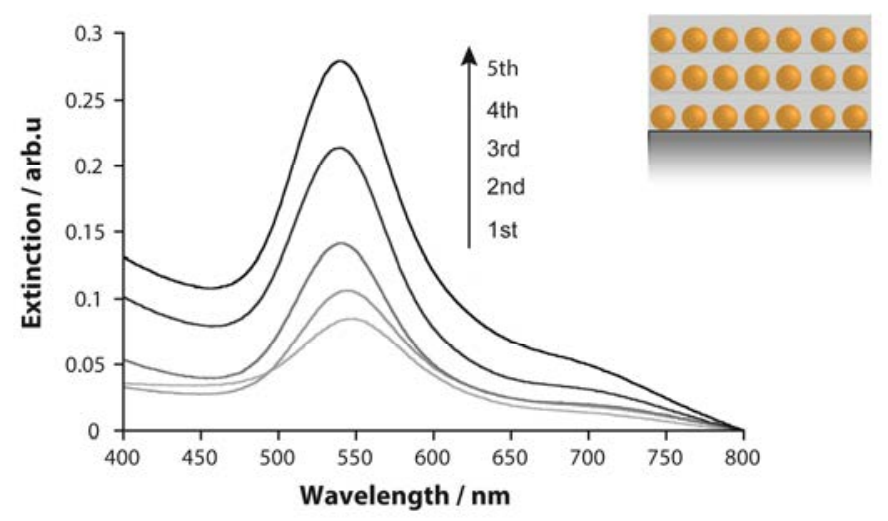

Figure 4.4: Light extinction increases when gold nanoparticle multilayers are formed. A rather thick polymer film made by spincoating was used to separate the nanoparticle layers. 


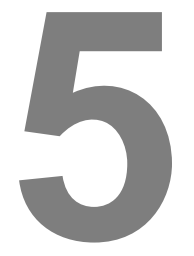

\section{Plasmonic biosensing}

\section{Biosensors - general introduction}

In general, a sensor is defined as a device that detects physical or chemical changes (e.g. temperature, pressure, light, concentration etc.) in our environment and converts them into measurable signals. An example of a historical sensor is the usage of canary birds in coal mines. They were used to detect the presence of carbon monoxide (CO), a toxic gas both for birds and humans. Since the birds are more sensitive than humans to lethal doses of $\mathrm{CO}$, the mine workers kept an eye on the bird while they were working and if the bird passed out, they knew it was time to get out of the mine. Today, this rather cruel method has been replaced with modern sensors which have significantly higher sensitivity and faster response time compared to the canary bird.

A biosensor is a specific type of sensor that utilizes a biological reagent in the sensing system. A frequently used definition of a biosensor is that it is a "selfcontained integrated device, which is capable of providing specific quantitative or semi-quantitative analytical information using a biological recognition element (biochemical receptor) which is retained in direct spatial contact with a 
transduction element”. ${ }^{118}$ However, in this definition, bioanalytical systems are not included and they are also considered to be biosensors by some. A broader definition would therefore be that it is "a tool used for analyzing biomolecular interactions". 119

The most successful and exploited biosensor is the glucose sensor. It is used daily by millions of diabetics all over the world to monitor their blood glucose level. The first glucose sensor was developed in 1962 by Clark and Lyon, using an enzyme-coated electrode. ${ }^{120}$ Modern glucose sensors are significantly smaller compared to the first prototype and are based on the enzyme glucose oxidase which generates a measurable current upon glucose oxidation. ${ }^{121}$ Biosensors are today important devices used in several different areas including industrial process monitoring, food quality control, drug development, environmental analysis and medical healthcare. Another classical example of a frequently used, commercially available biosensor is the home pregnancy test.

Biosensors are composed of two central components; a detector, consisting of biorecognition elements used for analyte identification, and a transducer which converts biological interactions into a measurable signal. Figure 5.1 is a schematic illustration of the components used in a biosensor.

The primary function of the biorecognition layer is to provide selectivity for the targets or analytes of interest. The biological elements incorporated in the biorecognition layer interact with specific analytes in the surrounding medium. Various classes of molecules have been exploited as biorecognition elements including enzymes, antibodies, nucleic acids, receptors etc. In addition to specificity, these elements need to possess several other important properties in order to assure a high performance of the biosensing device including stability, functionality, availability and, in some applications, also reversibility. 


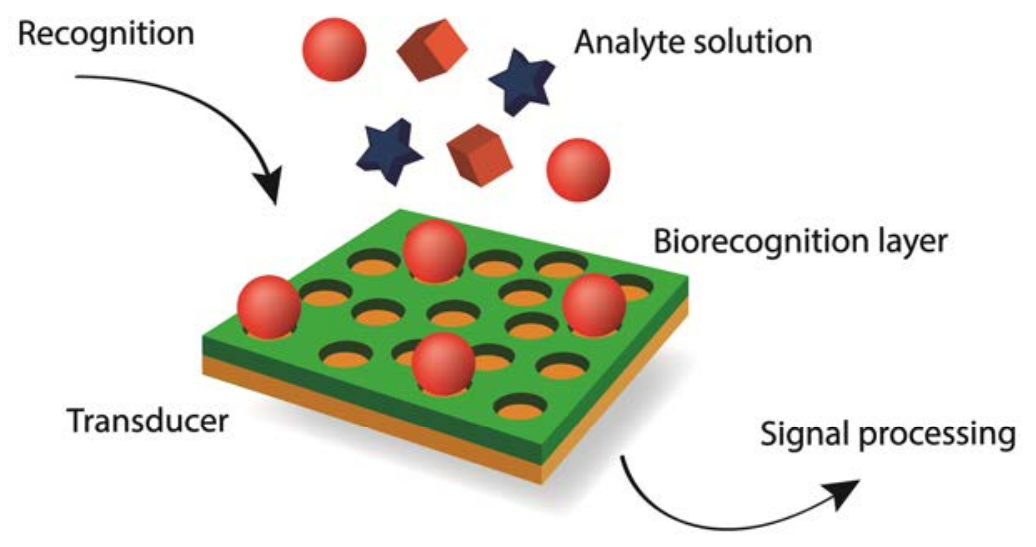

Figure 5.1: A schematic illustration of the components used in a biosensor. A biorecognition layer detects specific analytes from a complex solution and a transducer transforms the binding event to a measurable signal.

The transducer responds to the biochemical interaction and transforms it into a measurable signal. The choice of transduction mechanism party depends on the signal that is generated by the interaction between the biorecognition element and the analyte. A large variety of transduction mechanisms have been exploited in biosensors, including electrochemical, mechanical, thermal, and optical. If quantification is required, the signal output from the transducer should be correlated to the concentration of the analyte of interest where the concentration span depends on the dynamic range of the sensing system. Many biosensing systems can also provide information about the binding affinity and kinetics for the interaction.

The work included in this thesis focuses exclusively on optical transduction where visible light has been used for signal detection. Optical transducers offer a broad range of signal parameters that can be utilized for detection including the frequency, phase, polarization, and intensity of the light. Optical transduction techniques can be exploited for many applications and can generally be constructed by rather simple and inexpensive components. ${ }^{122,123}$ Optical fibers enable miniaturization and optical imaging can be used for multiplex analysis of arrays. 
Biosensors can be divided into different classes depending on: i) type of biochemical event, ii) the transduction mechanism or iii) the application area. However, they can also generally be divided into two groups, those that require labelling and those that do not. Label-free techniques such as SPR and Quartz Crystal Microbalance (QCM) enable a real-time, quantitative analysis of molecular binding events while label-based sensing requires tagging of the biomolecules in order to detect the interactions. Methods using labelling are often very sensitive, but a major drawback with these techniques is that a molecular labelling with, for instance, a fluorescent, enzymatic, or radioactive probe can affect the functionality of the molecule and induce molecular aggregation. For this reason there is an increased interest in label-free techniques for high-throughput screening of biomolecular interactions.

Most biosensors are based on the interaction of biomolecules bound to a solid support, since this facilitates washing, regeneration of the reagents, handling, and storage. Thus, an appropriate surface chemistry is of critical importance when producing biosensing devices with high performance. The surface chemistry should enable an immobilization of the biorecognition elements without affecting the functionality and at the same time be resistant to nonspecific adsorption. Much work has been invested into surface chemistry development, both with 2D-structures based on for example thiols ${ }^{124,125}$ or silanes ${ }^{126}$ and 3D-structures like hydrogels composed of various polymers (e.g. sugars and ethylene glycol). ${ }^{127,128}$ Hydrogels are particularly attractive since they can provide a suitable environment for the biomolecules, maintaining their structure and functionality. ${ }^{129}$

\section{SPR-based biosensing}

SPR-based sensing is one of the most exploited biosensing techniques and has been extensively used to monitor and characterize biomolecular interactions in real-time without the need for labelling. The first demonstration of SPR-based biosensing was performed by Liedberg et al. in 1983, where they studied the interaction between an antigen and an antibody on a silver surface. ${ }^{130}$ After their pioneering work, the technique was commercialized and is today routinely used 
by biochemists, pharmacologists and molecular biologists in order to determine biomolecular binding affinities and interaction kinetics. ${ }^{131}$

SPR-based sensors are based on refractometric detection using the excitation of a surface plasmon (see chapter 3). A typical setup for performing SPR biosensing experiments based on the Kretschmann configuration can be seen in Figure 5.2. In general, one interaction partner (ligand) is immobilized on a thin metal film, usually gold, followed by the introduction of the second interaction partner in solution (analyte). The analyte binding results in a change in the RI close to the metal surface, which changes the resonance condition for SP excitation. This can be monitored in real-time by measuring changes in the reflected light used for excitation. SPR-based sensors can be based on different interrogation modes including detection of angular, wavelength, intensity, or phase alterations. $^{131}$
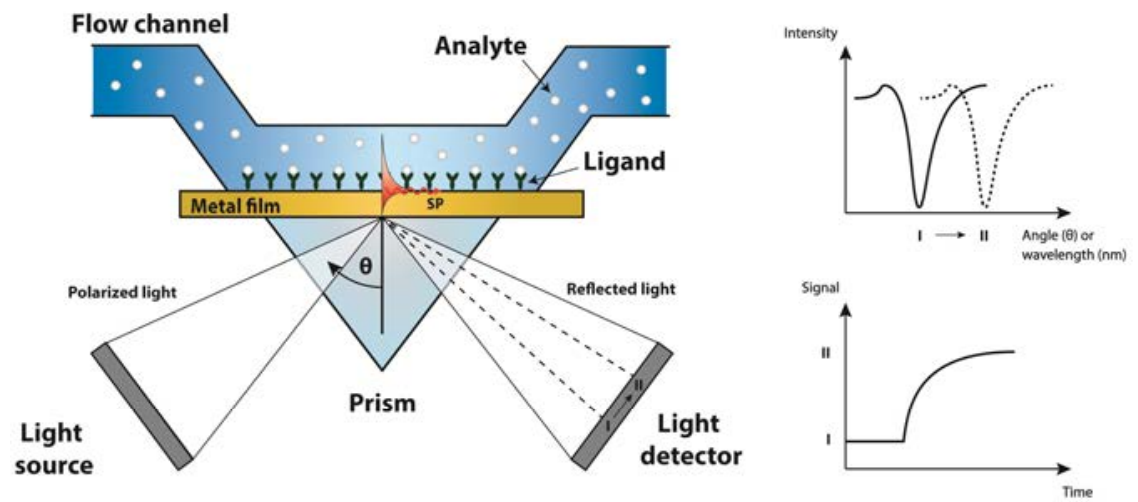

Figure 5.2: Setup for biosensing using SPR based on the Kretschmann configuration. Polarized light is guided through a glass prism and is totally internally reflected on the backside of a thin metal film creating an evanescent field through the metal film. At a specific angle or wavelength of the incident light the evanescent field can excite surface plasmons at the interface between the metal and the sample medium, resulting in a sharp decrease in the reflected light (upper right). If the refractive index changes at the metal interface the condition for SPR excitation is altered, thus changing the SPR angle or wavelength (from I to II in the figure). This allows for monitoring biomolecular events at the metal surface in real-time (lower right). 
SPR is generally considered to be a surface sensitive technique since the excitation of SPs creates an evanescent field that reaches out into the sample medium. The penetration depth is defined as the distance when the intensity of the electric field has decayed to about $1 / e$ of the value at the immediate surface. Any change of the RI within this region will alter the condition for SP excitation. The penetration depth depends on the excitation wavelength and the optical properties of the metal and the ambient medium and falls in the range of 150-300 $\mathrm{nm}$ in most SPR setups. This prompts a surface chemistry composed of a polymer 3D matrix which allows for an increased ligand surface concentration and utilization of the entire sensing volume. ${ }^{127}$

The overall performance of an SPR-based biosensor depends on several parameters including sensitivity, resolution, dynamic range, and limit of detection. The sensitivity is defined as the change in sensor response $(\delta Y)$ (angle, wavelength or intensity) caused by a change in the refractive index $(\delta n)$, which can be expressed as:

$$
\eta=\frac{\delta Y}{\delta n}
$$

The term $\eta$ is the RI sensitivity and this value depends on the choice of wavelength and the incident angle but is independent on the interrogation mode. The RI sensitivity can be divided into two different sensitivity expressions bulk and surface RI sensitivity. The bulk RI sensitivity $\left(\eta_{B}\right)$ refers to any RI changes in the entire sensing region. However, as SPR is routinely used to study molecular interactions at surfaces where only a fraction of the evanescent field is employed, it is also appropriate to introduce a term for the surface sensitivity $\left(\eta_{s}\right)$. This refers to the sensitivity for detecting a RI change $\left(\delta n_{d}\right)$ caused by the adsorption of a thin film of thickness $d$. The surface sensitivity term is especially relevant when comparing SPR with LSPR-based sensors, which is explored in

\section{Paper I.}




\section{LSPR-based refractometric biosensing}

Similar to conventional SPR-based sensing, nanostructures supporting a LSPR excitation can be used for refractometric sensing. As described in chapter 3, the condition for exiting electrons in metal nanostructures and creating localized surface plasmon resonance, depends on the dielectric properties of the surrounding medium. This allows for a spectroscopic detection of molecular interactions that occurs in the local proximity of the metal nanostructures (Figure 5.3). When the energy of the incident light matches the excitation energy of the electrons in the metal, a distinct peak known as the plasmon peak, can be seen in the optical extinction spectrum. By introducing molecules that induce a change of the RI in the surrounding environment, the resonance condition will be altered which results in a shift of the plasmon peak. The amount of material introduced around the metal nanostructures can be estimated by measuring the quantity of the plasmon peak shift.

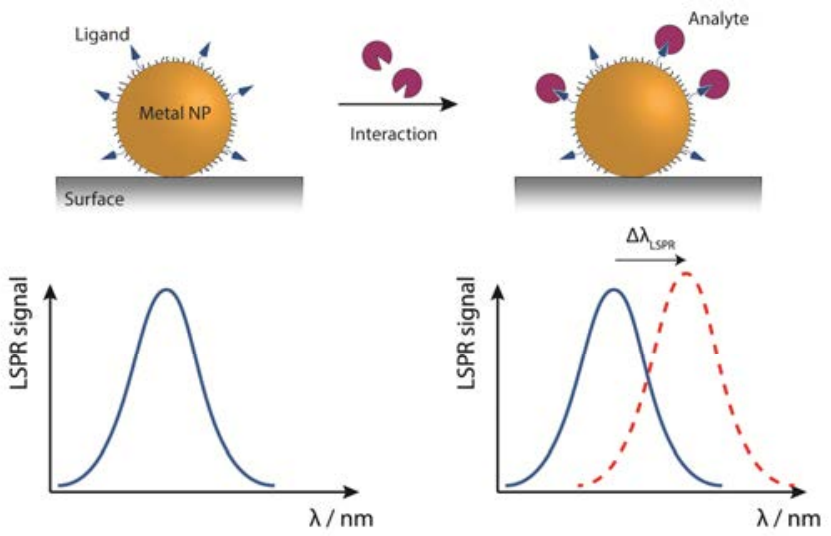

Figure 5.3: Schematic illustration showing the basic principle behind nanoplasmonic sensing based on refractometric detection. A shift of the plasmon peak is observed in the extinction spectra when an analyte interacts with a ligand on the surface of a metal nanoparticle. 
The first biosensing experiments using propagating surface plasmons were conducted in 1983 by Liedberg et al. ${ }^{130}$, but it took an additional 15 years before Englebienne demonstrated experimentally that also localized surface plasmons could be utilized for biosensing by detecting protein interactions using immobilized gold nanoparticles. ${ }^{132}$ Numerous of different LSPR-based sensing systems have been demonstrated after this, for detection of a large variety of biomolecular interactions such as biotin-streptavidin, ${ }^{133,134}$ DNA-DNA, ${ }^{135,136}$ antibody-antigen, ${ }^{27,28,132}$ etc.

The detection principle in LSPR-based refractometric sensing can be based on monitoring different spectral changes including intensity variations, either in the plasmon peak maxima or at a single wavelength, ${ }^{134,137,138}$ peak curvature changes, ${ }^{139}$ or by detecting the position of the plasmon resonance peak. ${ }^{27,28}$ The work included in this thesis focuses exclusively on the detection of plasmon peak wavelength shifts since this detection principle is favorable for randomly adsorbed nanoparticles onto substrates, where the surface coverage can vary somewhat across the surface. The surface coverage, i.e. the amount of particles used in the analysis will influence the intensity of the signal, but the plasmon peak position is not affected by the surface density of particles as long as there is no interparticle coupling occurring. However, this strategy demands an accurate determination of the spectral position of the plasmon peak maxima which requires a relatively high signal-to-noise ratio.

LSPR-based sensing show a great potential for miniaturized sensing and much work has been conducted on single nanoparticle detection, especially by the group of Van Duyne. ${ }^{140,141}$ Single nanoparticle spectroscopy has some advantages since it offers the ability to explore the optical properties of a specific nanostructure and how this is affected by the particle geometry. Also single nanoparticle detection enables multiplexed sensing schemes and a significant reduction of the absolute detection limit, which is required for achieving the ultimate sensitivity goal i.e. single molecule resolution. However, even if plasmonic nanostructures have large extinction cross sections the signal generated from a single nanoparticle is generally very weak which results in a low signal-to-noise ratio. Therefore, spectroscopic measurements on an ensemble of nanoparticles generally results in a better overall sensing performance. 
Refractometric sensing using localized surface plasmons is mainly focused on plasmonic nanostructures composed of either gold or silver. However, other materials such as aluminum, copper, platinum, and palladium, also display plasmonic properties which can be exploited for sensing applications. ${ }^{142-144}$ Recently, also non-metallic materials like semiconductor nanocrystals have been shown to exhibit plasmonic properties by introducing charge carriers through doping. ${ }^{145}$

\section{Refractive index sensitivity}

Over a narrow range of RI changes, the bulk RI sensitivity $\left(\eta_{B}\right)$ can be approximated as a linear function connecting the RI change $(\Delta n)$ and the LSPR peak shift $\left(\Delta \lambda_{\max }\right)$ :

$$
\Delta \lambda_{\max }=\eta_{B} \Delta n
$$

Thus, in order to maximize the sensing response i.e. the peak shift that occurs for a specific change in the surrounding RI, the bulk RI sensitivity should be as large as possible. Miller and Lazarides have shown using theoretical calculations that the bulk RI sensitivity for gold nanoparticles with a plasmon resonance below $800 \mathrm{~nm}$, solely depends on the plasmon peak position. ${ }^{146}$ Consequently, this indicates that even though the plasmon resonance depends on the shape and the size of the nanoparticles, the bulk RI sensitivity is not directly dependent on the particle geometry. Hence, two nanoparticles that give rise to an equivalent plasmon frequency but has totally different structures will exhibit a similar bulk RI sensitivity. Several studies have been reported with the aim of finding nanoparticles with high bulk RI sensitivities, ${ }^{30,147}$ but this has only been a quest for synthesizing nanoparticles displaying plasmon resonance bands at longer wavelengths. However, a longer resonance wavelength will not per se, give rise to a better sensing performance, which will be discussed in the next section. The correlation between the plasmon peak position and the bulk RI sensitivity has been verified experimentally by several groups including ours. ${ }^{147-149}$ In Paper IV, the RI sensitivity was investigated for a large variety of gold nanoparticles with 
different plasmon frequencies, obtaining a clearly linear relationship between the plasmon peak resonance position and the bulk RI sensitivity (Figure 5.4). However, it is important to notice that this correlation only is valid for dispersed nanoparticles in solution. Immobilization to a solid support or a surface functionalization will significantly affect the bulk RI sensitivity which will be addressed later in this chapter.

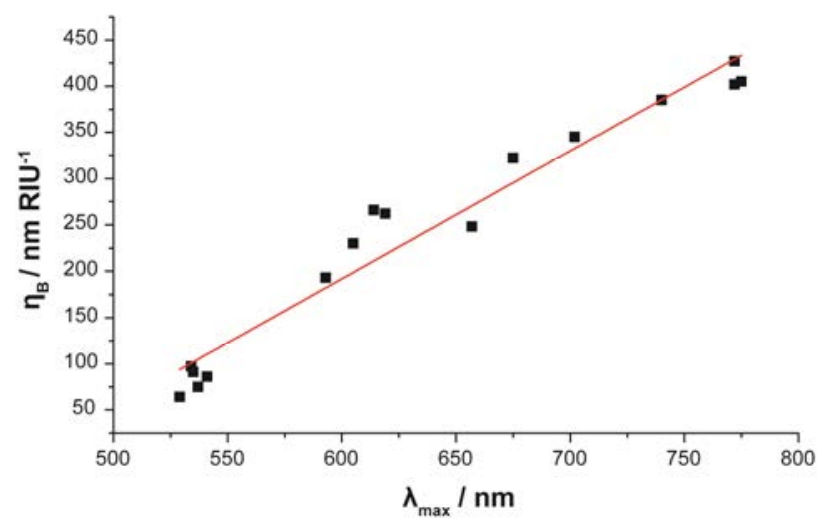

Figure 5.4: Experimentally obtained bulk RI sensitivity values for different types of gold nanoparticles plotted against the spectral position of their plasmon resonance peak. The straight line demonstrates the linear relationship between the two parameters.

Also, the correlation between the plasmon frequency and the bulk RI sensitivity is not applicable when comparing nanoparticles composed of different materials. For instance, silver nanoparticles exhibit plasmon bands located at shorter wavelengths compared to gold nanoparticles, but despite this fact, they exhibit higher bulk RI sensitivities. ${ }^{150}$ This can be explained by the different dielectric properties of the two materials. Despite favorable RI sensitivities, silver nanoparticles suffer from chemical instability both in air and aqueous environments which limits their practical use.

Table 5.1 summaries some of the experimentally obtained properties of all metal nanoparticle structures used in the work included in this thesis. The highest bulk RI sensitivity is seen for gold-coated silver nanoprisms which were used in Paper I. 
Table 5.1: Summary of the physical properties of the metal nanoparticles used in the work included in this thesis.

\begin{tabular}{|c|c|c|c|c|c|}
\hline & Geometry & $\begin{array}{l}\text { Size } \\
(\mathrm{nm})\end{array}$ & $\begin{array}{c}\Lambda_{\text {LSPR }} \\
(\mathrm{nm})\end{array}$ & $\begin{array}{c}\eta_{B} \\
(\mathbf{n m} / \mathbf{R I U})\end{array}$ & FOM \\
\hline O & Gold nanosphere & 25 & 525 & 60 & 1.2 \\
\hline & Gold nanosphere & 50 & 532 & 80 & 1.5 \\
\hline & Gold nanosphere & 100 & 578 & 180 & 1.6 \\
\hline & $\begin{array}{l}\text { Gold-coated silver } \\
\text { nanoprism }\end{array}$ & $30-40$ & 660 & 425 & 3.0 \\
\hline & Silver nanosphere & 35 & 398 & 144 & 3.0 \\
\hline & Silver nanocube & 40 & 444 & 158 & 3.3 \\
\hline 0 & Silver nanoplate & $30-40$ & 552 & 315 & 3.6 \\
\hline & Gold nanocube & 40 & 535 & 91 & 2.0 \\
\hline & $\begin{array}{l}\text { Concave gold } \\
\text { nanocube }\end{array}$ & 50 & 615 & 266 & 2.6 \\
\hline & Gold nanorod & $65 \times 20$ & 740 & 385 & 3.6 \\
\hline & Gold nanosprism & $50-60$ & 775 & 405 & 3.8 \\
\hline
\end{tabular}




\section{Figure of Merit (FOM)}

As plasmonic materials give rise to peak features in their extinction spectrum, it is convenient to analyze the sensing data based on the spectral changes that occurs for these peaks. However, since spectroscopic measurements only provide the relative extinction intensity of light at different wavelengths, the discrete data points acquired needs to be converted into a parameter that accurately can represent the sensing result. ${ }^{77}$. If the intensity is measured and compared at a single wavelength the post signal processing required is rather small, but normally it is necessary to include a larger spectral region in the data analysis. As discussed in the previous section, the RI sensitivity is defined by detecting either the wavelength or the intensity shift of the plasmon peak maxima that occurs upon a change in the RI close to the nanostructures. However, a determination of the plasmon peak maxima requires a mathematical calculation that depends on the width of the plasmon peak and the intensity of the signal, or more accurately the noise level in the signal. It is therefore necessary to introduce a term that includes resolution in order to make a fair comparison between different plasmonic nanostructures since the peak width can vary significantly. This has motivated the introduction of a new parameter known as figure of merit (FOM) that includes both sensitivity and resolution. FOM is defined as the ratio between the bulk RI sensitivity and the peak width:

$$
\mathrm{FOM}=\frac{\eta_{B}}{f w h m}
$$

where fwhm is an acronym for full-width-half-maximum and is defined as the width (in $\mathrm{nm}$ ) of the plasmon peak at half of its maximum value. Calculated FOM values for all nanoparticles used within this work can be seen in Table 5.1.

As discussed in the previous section, the bulk RI sensitivity generally depends on the spectral position of the plasmon resonance peak. However, shifting the LSPR peak position to longer wavelength into the infrared (IR) region can result in a significant broadening of the plasmon peak due to dephasing and radiative damping effects. Consequently, an increase in the FOM value is not expected for 
longer wavelengths since the higher bulk RI sensitivity will be compensated by a broader plasmon peak i.e. a larger fwhm value. Thus, tuning the LSPR position into the IR region will increase the sensitivity but reduce the resolution and will therefore not give any additional improvements of the sensing performance. This is also attractive for practical reasons since relocation into the IR region requires the usage of more advanced optical equipment.

Motivated by the issue discussed above, Otte and Sepulveda calculated the optimum spectral region where the FOM value is maximized. ${ }^{77,151}$ They used the dipolar approximation to calculate the optical response and RI sensitivity of ellipsoidal gold nanoparticles, by varying the aspect ratio but keeping the volume constant. They obtained, unsurprisingly, a nearly linear increase of the bulk RI sensitivity with increasing wavelength of the LSPR peak position (Figure 5.5 A). However, a distinct minimum was observed for the fwhm value at $\lambda_{\max } \sim 700 \mathrm{~nm}$. By calculating the ratio between the sensitivity and the fwhm values they obtained maximum FOM values in the spectral region comprised between 700 and $900 \mathrm{~nm}$ (Figure $5.5 \mathrm{~B}$ ). These calculations clearly show that longer resonance wavelengths can result in lower FOM values, hence a reduction of the sensing performance.
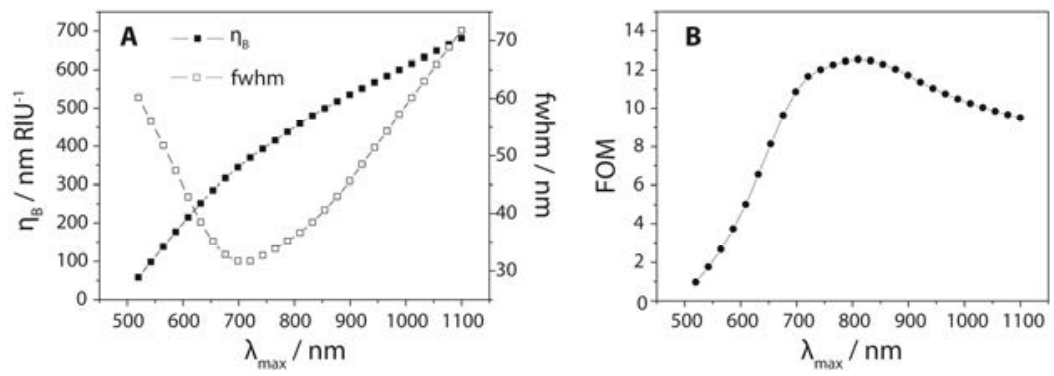

Figure 5.5: (A) Wavelength dependence of the bulk RI sensitivity and the fwhm value for ellipsoidal gold nanoparticles with different aspect ratio. (B) Wavelength dependence of the FOM value calculated by the ratio between the bulk RI sensitivity and the fwhm value. Data kindly provided by Dr. Borja Sepulveda (CIN2, Barcelona) 


\section{Substrate effect}

Since refractometric sensing is solely based upon the detection of RI changes, any variation of the RI within the sensing volume will give rise to changes in the output signal used for detection. Thus, in order to maximize the sensitivity the effective RI change caused by, for instance, the introduction of a biomolecule inside the sensing volume, should be as large as possible. Consequently, a larger response will be attained by either detecting molecules that gives a larger RI response or by decreasing the RI of the surrounding medium within the sensing volume before introducing the target molecules. For example, the addition of a biomolecular layer ( $n \sim 1.46)$ that occupies the entire sensing volume will give a 3.5 times higher response, if air ( $n \sim 1.0)$ is the surrounding medium rather than water ( $n \sim 1.33)$, assuming a linear response. However, for practical reasons, biomolecular detection is normally carried out in liquid. The largest response possible is thus achieved when the ambient medium in the vicinity of the nanoparticle surface is completely replaced by the target molecules.

As discussed in chapter 4, tethering nanoparticles to a solid support has many advantages when it comes to detecting biomolecular interactions. However, one obvious disadvantage is that the substrate will reduce the RI sensitivity in a nanoplasmonic refractometric sensing system. When introducing a substrate in the direct vicinity of the metal nanoparticles it will occupy a certain space within the sensing volume of the nanoparticles thereby eliminating the ability to detect any RI changes within this region. Hence, the substrate influence on the RI sensitivity depends on how much space the substrate occupies. This is known as the substrate effect and it is connected with the surface area of the nanoparticles that is in direct contact with the underlying substrate (Paper III and IV). 
The substrate effect can be estimated using the effective medium approximation which is used for calculating the effective RI value $\left(n_{e f f}\right)$ around a structure with a specific material like for instance a metal nanoparticle.

$$
n_{e f f}=X_{A} n_{s}+\left(1-X_{A}\right) n_{d}
$$

where $X_{A}$ is the fractional surface area connected to the substrate and $n_{s}$ and $n_{d}$ is the RI of the substrate and the surrounding medium, respectively. This is schematically illustrated in Figure 5.6 where a gold nanocube is immobilized onto a substrate where one side of the nanocube (1/6) is in direct contact with the substrate i.e. $X_{A}=0.167$. Thus, the bulk RI sensitivity of a metal nanocube is expected to be reduced around $17 \%$ upon a surface immobilization using the effective medium approximation. Sensitivity experiments verify that this approximation is an effective way to estimate the surface effect on the bulk RI sensitivity of metal nanoparticles (Paper III and IV). However, it should be noted that this approximation is not that good for estimating the surface effect for complex, anisotropic nanoparticles and for nanoparticle batches with high polydispersity.

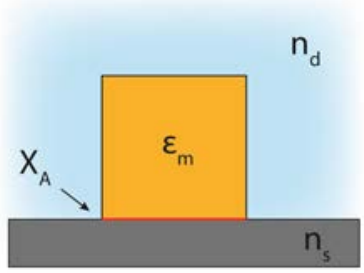

Figure 5.6: A schematic illustration of a gold nanocube immobilized on a solid support with a surface contact fractional area $\boldsymbol{X}_{\boldsymbol{A}}$. 
If the material of the substrate has a higher RI value than the surrounding medium $\left(n_{s}>n_{d}\right)$ the plasmon resonance peak position will be red-shifted to a longer wavelength since the effective RI around the nanoparticles will increase. The quantity of this shift will also depend on the fractional surface area in contact with the substrate since this will affect the effective RI around the particles.

In addition, the introduction of a substrate with a higher RI value can cause a redistribution of the electromagnetic field around the nanoparticles, shifting it towards and into the substrate. ${ }^{152,153}$ This will increase the substrate effect on the sensitivity since RI changes cannot be detected within this electromagnetic field. However, this effect can be minimized by decreasing the RI of the underlying substrate since a larger portion of the electromagnetic field then will be comprised in an accessible sensing region. Brian et al. showed that the substrate effect can be reduces for nanoplasmonic structures using substrates made by Teflon which has a low RI value $(n=1.32) .{ }^{154}$

Another obvious effect caused by nanoparticle immobilization onto a substrate is that the surface accessible for biofunctionalization on the nanoparticles reduces i.e. the maximum amount of recognition molecules is decreased. Consequently, this affects the overall sensing performance since a lower quantity of analytes can interact with the capturing molecules within the sensing region thereby reducing the maximum response.

So in conclusion, in order to achieve maximum sensitivity in refractometric sensing systems based on surface-bound nanoplasmonic material the contact area with the underlying substrate should be minimized and the RI of the substrate should be as low as possible. The substrate effect for various metal nanoparticles has been explored in both Paper III and Paper IV. 


\section{Sensing volume}

So far, only the bulk RI sensitivity of nanoparticles has been discussed which concerns RI changes within the entire sensing volume. However, in many sensing applications only a fraction of the sensing volume is exploited. Therefore, it is essential to also consider how the RI sensitivity varies with the distance from the metal surface i.e. how the electromagnetic field propagates into the ambient medium. For metal nanoparticles, the electromagnetic field created upon excitation is highly localized to the proximity of the nanoparticles where the decay length $(L)$ is considered to be around $\sim 10-20 \mathrm{~nm}^{155,156}$ (Figure 5.7). However, it is important to notice that the field distribution can vary significantly between different nanostructures but also around a single nanoparticle. ${ }^{45,157}$ It has been shown that the RI sensitivity can be extensively increased in areas where the electromagnetic field is enhanced. ${ }^{133,158,159}$ Thus, if molecular detection can be addressed to these specific "hot spots" the overall sensitivity could be significantly improved. However, this is very complicated to implement in practice.

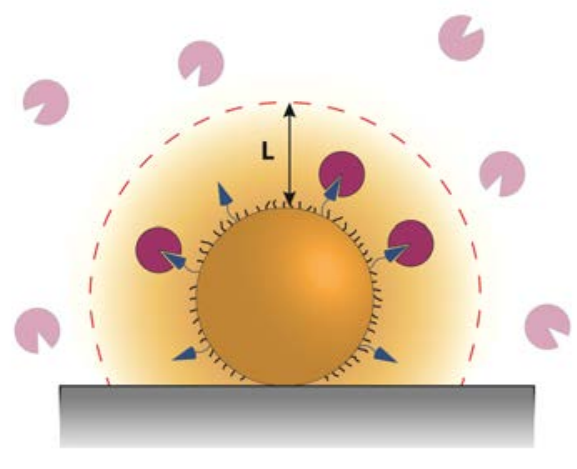

Figure 5.7: The sensing volume is highly localized to the vicinity of the metal nanoparticles and depends on the decay length $(L)$ of the electromagnetic field. 
Instead of trying to quantify the binding of a specific molecule it is much more convenient to consider the adsorption of a single molecular layer to the surface of a metal nanostructure. If the electromagnetic field is assumed to decay exponentially from the particle surface, the spectral wavelength shift that occurs when a thin film is added to a metal nanostructure can be estimated using the following expression:

$$
\Delta \lambda_{\max }=\eta_{B} \Delta n\left(1-e^{-\frac{2 d}{L}}\right)
$$

where $\eta_{B}$ is the bulk RI sensitivity, $\Delta n$ is the difference in RI between the ambient medium and the adsorbed material, $d$ is the thickness of the adsorbed film, and $L$ is the decay length of the electromagnetic field. This expression is largely determined based on empirical measurements, but it provides a decent approximation of the maximum sensing response that can be obtained upon a molecular adsorption. From Equation 5.5 it is obvious that the peak shift can be maximized by increasing either the bulk RI sensitivity or the induced RI change and by optimizing the thickness of the adsorbed film and the decay length of the electromagnetic field.

Since the actual decay length of the electromagnetic field is difficult to determine experimentally it is more appropriate to report about the sensing distance i.e. the average distance from the surface of a metal nanostructure where a change in the RI can be detected. The sensing distance from numerous metal nanostructures has been explored experimentally using a variety of surface layer coatings including polymers, ${ }^{160-162}$ silica, ${ }^{163,164}$ or alkanethiols. ${ }^{155,165}$ all reporting a sensing distance of around 20-30 nm. Hence, molecules further away than the sensing distance, i.e. outside the sensing volume, will not cause a shift of the plasmon peak resonance position. So in order to maximize the sensing response, the sensing volume should be optimized so it only includes the molecular events that are supposed to be detected. 
In Paper I, polyelectrolyte layers were used to compare the sensing distance between metal nanoparticles and conventional SPR based on propagating surface plasmons. It is shown that the sensing distance for LSPR is significantly smaller compared to SPR (by roughly one order of magnitude) however, the sensitivity for detecting RI changes close to the metal surface can actually be higher for LSPR-based sensors compared to sensors based on SPR. Others have report on similar sensing performance between the two sensing schemes when it comes to detecting molecular events close to the metal surface. ${ }^{166}$ The performance is comparable since the sensing volume around the nanostructures better matches the volume of the molecular interaction compared to ordinary SPR-based sensing, even though the bulk RI sensitivity is significantly higher for propagating plasmons compared to localized plasmons.

Detection of small analytes with low molecular weights is problematic in conventional SPR-based sensing since the induced RI change is so small compared to the large background signal, thus a careful sample preparation and background subtraction is extremely important in order to attain a detectable response. As the sensing volume in LSPR-based sensing system is evidently small, the background signal, also known as the bulk response, can be significantly reduced as compared to SPR-based systems. Sensing based on LSPR thus show a higher potential for detecting small molecules since the bulk contribution is considerably lower and the induced RI change is not obscured by the background noise. For example, Kabashin et al. were able to detect biotin (244 Da) instead of Streptavidin, using localized surface plasmons created in gold nanopillars. ${ }^{167}$ Also, studies of protein conformational changes has been demonstrated using LSPR detection. ${ }^{168}$

The highly localized sensing volume around metal nanostructures is not only advantageous since this limits the usage of larger recognition molecules as well as the usage of various surface functionalization chemistries such as the wellestablished dextran matrix often used in SPR-based biosensors. ${ }^{127}$ For example, 
when introducing one of the most frequently used biorecognition elements i.e. antibodies, to the surface of a metal nanoparticle, they will occupy a large fraction of the sensing volume thereby reducing the analyte sensitivity significantly. Thus, it is preferable to use small biorecognition elements, like antibody fragments or peptides in LSPR-based sensing in order to fully exploit the small sensing region around the metal nanostructures.

A sensing device produced for detecting specific analytes in a complex media needs to resistant towards undesired molecular interactions. There are typically three different types of interactions that needs to be avoided, i) unspecific binding of other molecules present in the sample directly to or in the close proximity of the nanoparticle surface, ii) binding of other molecules to the biorecognition element i.e. problem with specificity and iii) the analyte of interest can itself bind unspecifically direct or close to the particle surface. All of the binding events described above will result in a false positive response. Thus, minimizing undesired molecular binding is crucial in order to attain a high sensing performance. However, due to the small sensing volume around plasmonic nanostructures, the possibility for incorporating various surface chemistries that exhibit low non-specific binding e.g. PEG chains or a dextran matrix is highly limited since such a modification will occupy almost the entire sensing volume. Consequently, this will reduce the ability for detecting specific target molecules. Functionalizing nanostructures with a surface chemistry that both exhibit specificity and at the same time has a resistance towards nonspecific binding of other molecules is thus a great challenge. 


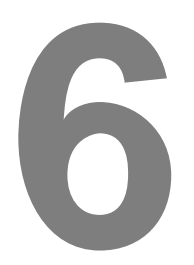

\section{Summary of papers}

Four papers are included in this thesis. Three has been published in peer-review journals and one is in manuscript.

This chapter provides a short summary of the included papers. 


\section{Paper I}

\section{Local Refractive Index Sensing Based on Edge Gold-Coated Silver Nanoprisms}

Martinsson E, Shahjamali MM, Enander K, Boey F, Xue C, Aili D and Liedberg B.

Journal of Physical Chemistry C 2013, 117, 23148-23154

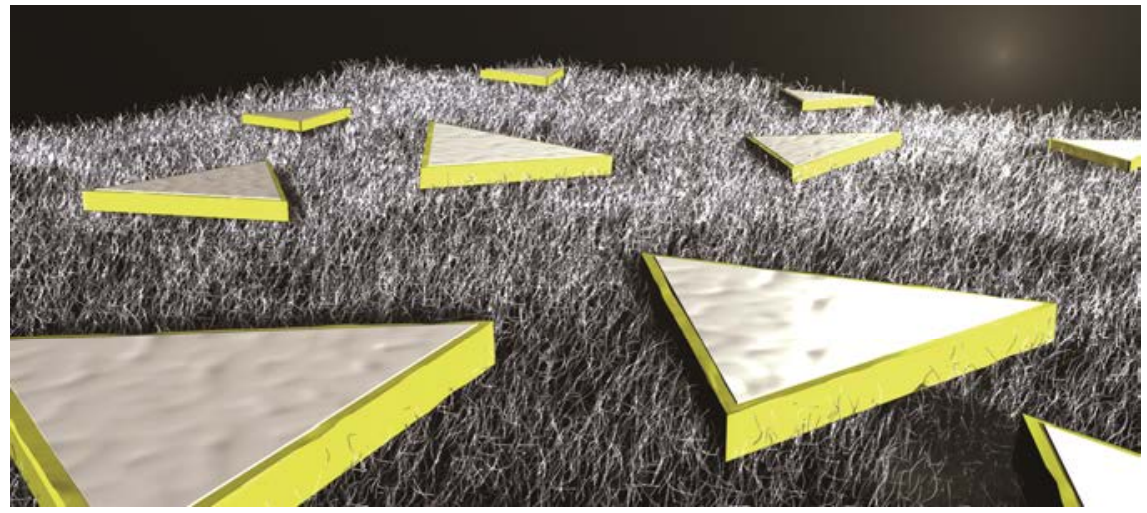

This paper examines the bulk and local RI sensitivity of edge gold-coated silver nanoprisms compared to conventional SPR and spherical gold nanoparticles. Silver nanoprisms were synthesized using a photo-mediated synthesis protocol followed by a gold coating which protects the sharp-tip silver features from oxidation. A layer-by-layer assembly of polyelectrolytes, which enables a nanometer thickness control of each deposited polyelectrolyte layer and protein adsorption, was used to examine the local RI sensitivity. The results shows that the gold-coated silver nanoprisms exhibits a four-fold higher local RI sensitivity compared to conventional SPR despite a significantly lower bulk RI sensitivity and eight times higher sensitivity compared to spherical gold nanoparticles. These hybrid gold-coated silver nanoprisms thus show a great potential for detecting small local RI changes which is a highly attractive characteristic for transducer elements in LSPR-based biosensing devices based on refractometric detection. 


\section{Paper II}

Optimizing the Refractive Index Sensitivity of Plasmonically Coupled Gold Nanoparticles

Martinsson E, Sepulveda B, Chen P, Elfwing A, Liedberg B and Aili D.

Plasmonics 2014, 9, 773-780

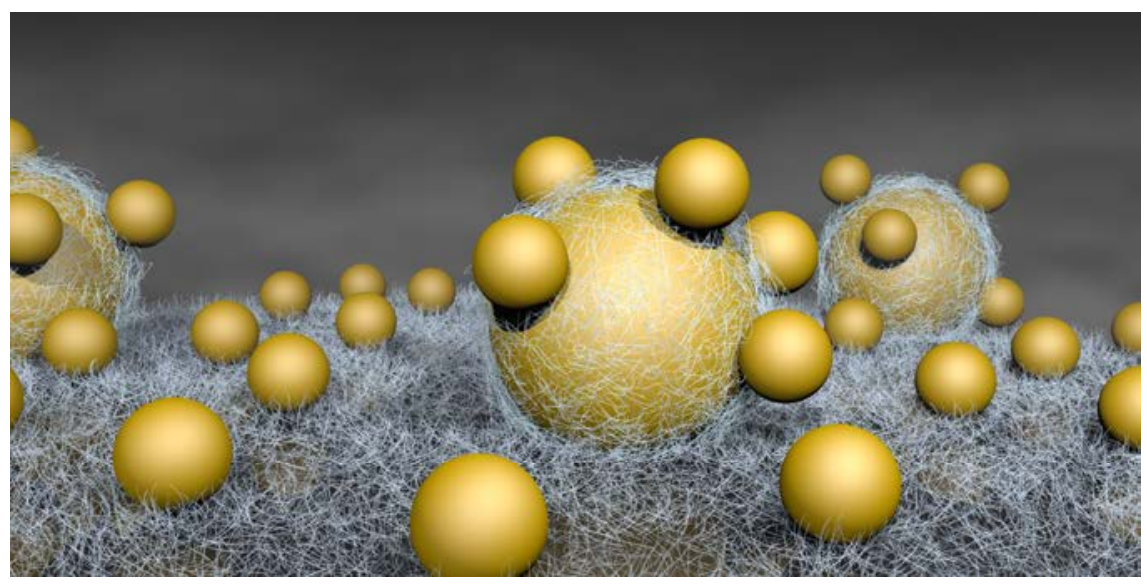

Herein, a protocol for controlling the self-assembly and separation of spherical gold nanoparticles has been developed in order to explore the plasmonic coupling effect on the RI sensitivity. Polyelectrolyte layers were used to control the interparticle distance between spherical gold nanoparticles of different sizes. A strong coupling was observed between the gold particles generating distinct plasmon peaks where the frequency could be tuned by varying either the size of the nanoparticles or the interparticle spacing. Examination of the bulk RI sensitivity showed a significantly higher sensitivity for plasmonic coupling modes compared to plasmon bands from single nanoparticles. The presented strategy is simple and scalable and increases the rather modest RI sensitivity of spherical gold nanoparticles. 


\section{Paper III}

\section{Substrate Effect on the Refractive Index Sensitivity of Silver Nanoparticles}

Martinsson E, Otte MA, Shahjamali MM, Sepulveda B and Aili D.

Journal of Physical Chemistry C 2014, In Press

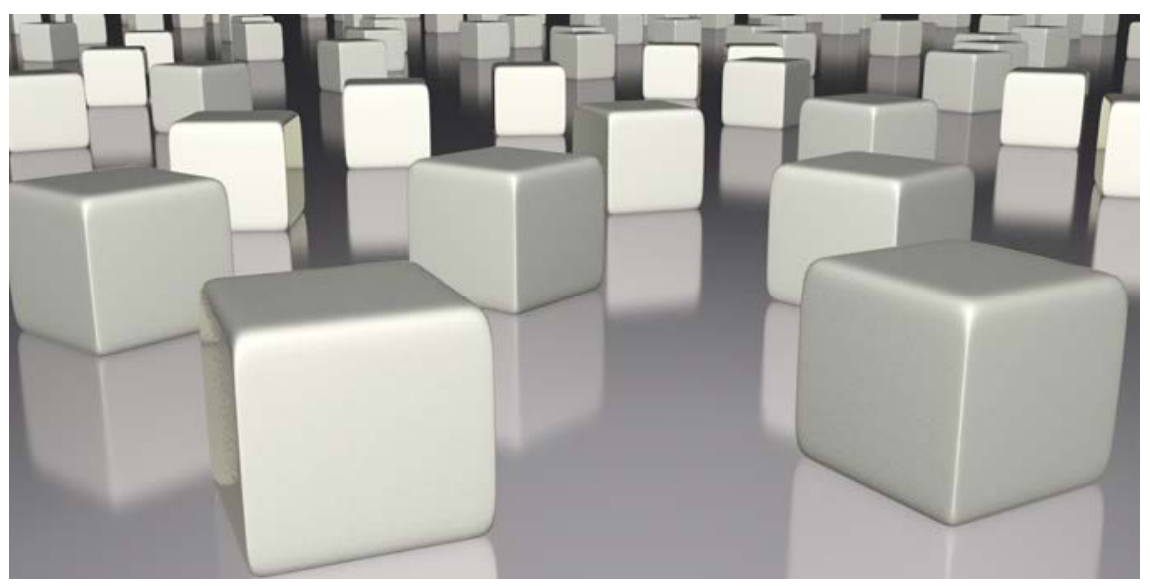

The aim of this paper was to explore how the RI sensitivity is affected when metal nanoparticles are immobilized on a solid support. Silver nanoparticles with three different shapes (spheres, cubes and plates) were chemically synthesized. The bulk RI sensitivity of dispersed nanoparticles was determined and compared to the sensitivity of immobilized nanoparticles. Both experimental and theoretical results showed a strong correlation between the fractional surface area in direct contact with the underlying substrate and the reduction of the RI sensitivity. Nanoplates showed the largest decrease in sensitivity (-36\%) upon immobilization, drastically reducing their advantage over other nanoparticles. This works shows that the substrate effect is important to consider when developing nanoplasmonic sensing devices based on metal nanoparticles. 


\section{Paper IV}

Influence of Surfactant Bilayers and Substrate Immobilization on the Refractive Index Sensitivity of Anisotropic Gold Nanoparticles

Martinsson E, Shahjamali MM, Large N, Zaraee N, Schatz GC, Mirkin CA and Aili D.

2014, In Manuscript

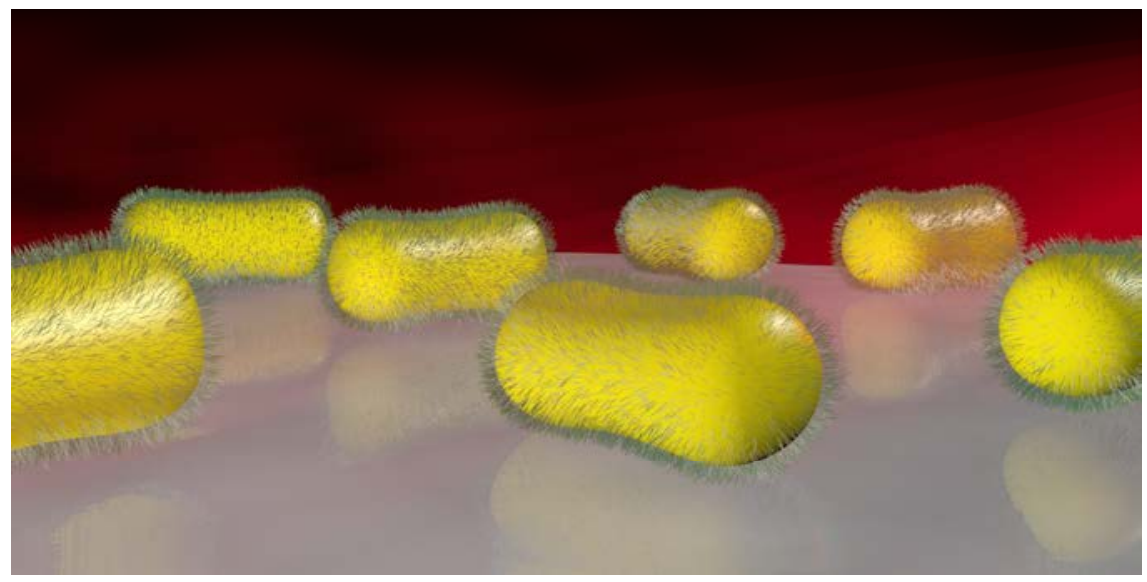

This work examines the influence of surfactant bilayers and substrate immobilization on the RI sensitivity of gold nanoparticles. Surfactants like CTAX are commonly used to control the shape of gold nanoparticles. During synthesis, the gold nanoparticles are covered with a 3-4 nm thick bilayer of CTAX, which significantly affects their possibility to detect local RI changes. In this work, four types of gold nanoparticles with different geometry were synthesized using CTAX. Their bulk RI sensitivity was examined both theoretically and experimentally as well as the effect of a substrate immobilization. In addition, it is shown that the RI sensitivity can be significantly increased by exposing the CTAX-covered nanoparticles to a short (30 sec) oxygen plasma treatment, where some of the surface-bound CTAX is etched away from the surface. A longer plasma exposure (30 min), however, leads to a reduction in the RI sensitivity caused by morphological changes of the nanoparticles. It is shown that a removal of CTAX using oxygen plasma treatment can increase the RI sensitivity with up to $40 \%$. 


\section{Future perspective}

Metal nanoparticles are indeed promising transducer elements for biosensing devices based on refractometric detection. Localized surface plasmons can be excited by light in the visible or near-infrared wavelength range and highly sensitive devices can be constructed using rather simple instrumentation. Therefore, LSPR-based methods are especially suitable for miniaturized sensors in, for example, point-of-care diagnostics or in other applications outside core lab facilities. However, much work is required before they can be practically implemented and utilized in biomolecular sensing. Several challenges need to be addressed in order to realize LSPR-based sensing devices that are sensitive, specific, and robust, while also being cost-effective. Some of these challenges are discussed below:

Biofunctionalization: A reliable and biofunctional surface chemistry is a key aspect in order to produce a successful biosensing device that enables molecular recognition in complex samples. The surface chemistry should exhibit a high selectivity as well as a satisfactory resistance against unspecific adsorption. To compress all these functionalities onto the surface of nanoparticles without 
significantly reducing their sensitivity is thus a great challenge and will require a substantial combined research effort. Focus should be on the development of thin surface coatings that display low non-specific adsorption together with the production of small recognition elements that provides specificity without occupying the entire sensing volume.

Reproducibility: The techniques for fabrication of metal nanostructures have advanced significantly during the last decade, but there is room for improvement in this area especially when it comes to reproducibility. Nanostructures made by various lithography techniques can yield high reproducibility, although generally at higher production costs and with problems with up-scaling. Self-assembly of nanoparticles made by wet-synthesis strategies offers a simple and cost effective way to produce nanoplasmonic substrates. However, the reproducibility needs to be improved, in particular the polydispersity in the size and shape of the synthesized nanoparticles and thereby obtaining narrow plasmon bands. Also, a better control of the self-assembly process is critical in order to produce substrates with identical surface nanoparticle coverages that give rise to similar intensities in the output signal. This could be achieved by combining selfassembly with soft lithography techniques in order to guide the surface adsorption of the nanoparticles. This will also enable the creation of nanopatterns which is important for multiplexed detection.

Stability: Nanoparticle robustness is another key aspect to consider when developing sensing devices with reliable output signals. Morphological changes of the metal nanoparticles can give rise to spectral changes that can interfere with the sensing response and hence, produce negative results. The possibility to introduce various chemical and biological substances in the close proximity of metal nanoparticles without affecting their structure is of critical importance.

Localization: A higher sensing performance can be achieved by controlling the orientation of nanoparticles since the sensitivity for detecting local RI changes around or in between anisotropic nanoparticles can be significantly improved if the molecular interactions can be guided into areas with enhanced electromagnetic fields. Thus, it is better to focus on the nanoparticle assembly rather than trying to optimize the structure of individual nanoparticles since a good control of the interparticle separation and orientation will provide higher 
sensitivities compared to single nanoparticles. It is, however, a great challenge to simultaneously control the immobilization of recognition molecules into these areas with enhanced sensitivity.

The work presented in this thesis provides comprehensive information about how metal nanoparticles can be utilized for nanoplasmonic sensing, especially regarding nanoparticle immobilization and optimization of the RI sensitivity. However, several challenges remain in order to fully demonstrate their potential as sensing transducers and take the next step in the development. A joint multidisciplinary effort is undoubtedly needed in order to produce nanoplasmonic sensing platforms that could compete with existing sensing techniques. 


\section{References}

1. Feynman, P. R., There's Plenty of Room at the Bottom. Caltech Engineering and Science 1960, 23, 22-36.

2. Stockman, M. I., Nanoplasmonics: past, present, and glimpse into future. Optics Express 2011, 19, 22029-22106.

3. Giannini, V.; Fernandez-Dominguez, A. I.; Sonnefraud, Y.; Roschuk, T.; Fernandez-Garcia, R.; Maier, S. A., Controlling Light Localization and Light-Matter Interactions with Nanoplasmonics. Small 2010, 6, 2498-2507.

4. Hutter, E.; Fendler, J. H., Exploitation of localized surface plasmon resonance. Advanced Materials 2004, 16, 1685-1706.

5. Willets, K. A.; Van Duyne, R. P., Localized surface plasmon resonance spectroscopy and sensing. Annual Review of Physical Chemistry 2007, 58, 267-297.

6. Lal, S.; Link, S.; Halas, N. J., Nano-optics from sensing to waveguiding. Nature Photonics 2007, 1, 641-648.

7. Brill, R. H., The Chemistry of the Lycurgus Cup. Proc 7th Internat. Cong. Glass, comptes rendus 2 1965, Paper 223, 1-13.

8. Barber, D. J.; Freestone, I. C., An investigation of the origin of the color of the lycurgus cup by analytical transmission electron-microscopy. Archaeometry 1990, 32, 33-45.

9. Hornyak, G. L.; Patrissi, C. J.; Oberhauser, E. B.; Martin, C. R.; Valmalette, J.-C.; Lemaire, L.; Dutta, J.; Hofmann, H., Effective Medium Theory Characterization of Au/Ag Nanoalloyporous Alumina Composites. NanoStructured Materials 1997, 9, 571-574.

10. Maier, S. A.; Brongersma, M. L.; Kik, P. G.; Meltzer, S.; Requicha, A. A. G.; Atwater, H. A., Plasmonics - A route to nanoscale optical devices. Advanced Materials 2001, 13, 1501-1505.

11. Barnes, W. L.; Dereux, A.; Ebbesen, T. W., Surface plasmon subwavelength optics. Nature 2003, 424, 824-830.

12. Maier, S. A.; Kik, P. G.; Atwater, H. A., Observation of coupled plasmon-polariton modes in Au nanoparticle chain waveguides of different lengths: Estimation of waveguide loss. Applied Physics Letters 2002, 81, 1714-1716.

13. Atwater, H. A.; Polman, A., Plasmonics for improved photovoltaic devices. Nature Materials 2010, 9, 205-213.

14. Wu, J. L.; Chen, F. C.; Hsiao, Y. S.; Chien, F. C.; Chen, P. L.; Kuo, C. H.; Huang, M. H.; Hsu, C. S., Surface Plasmonic Effects of Metallic Nanoparticles on the Performance of Polymer Bulk Heterojunction Solar Cells. Acs Nano 2011, 5, 959-967.

15. Pillai, S.; Catchpole, K. R.; Trupke, T.; Green, M. A., Surface plasmon enhanced silicon solar cells. Journal of Applied Physics 2007, 101, 8. 
16. Christopher, P.; Xin, H. L.; Linic, S., Visible-light-enhanced catalytic oxidation reactions on plasmonic silver nanostructures. Nature Chemistry 2011, 3, 467-472.

17. Langhammer, C.; Larsson, E. M.; Kasemo, B.; Zoric, I., Indirect Nanoplasmonic Sensing: Ultrasensitive Experimental Platform for Nanomaterials Science and Optical Nanocalorimetry. Nano Letters 2010, 10, 3529-3538.

18. Anker, J. N.; Hall, W. P.; Lyandres, O.; Shah, N. C.; Zhao, J.; Van Duyne, R. P., Biosensing with Plasmonic Nanosensors. Nature Materials 2008, 7, 442-453.

19. Jain, P. K.; Huang, X. H.; El-Sayed, I. H.; El-Sayed, M. A., Noble Metals on the Nanoscale: Optical and Photothermal Properties and Some Applications in Imaging, Sensing, Biology, and Medicine. Accounts of Chemical Research 2008, 41, 1578-1586.

20. Sepulveda, B.; Angelome, P. C.; Lechuga, L. M.; Liz-Marzan, L. M., LSPR-based nanobiosensors. Nano Today 2009, 4, 244-251.

21. Haes, A. J.; Haynes, C. L.; McFarland, A. D.; Schatz, G. C.; Van Duyne, R. R.; Zou, S. L., Plasmonic materials for surface-enhanced sensing and spectroscopy. Mrs Bulletin 2005, 30, 368-375.

22. Yonzon, C. R.; Stuart, D. A.; Zhang, X. Y.; McFarland, A. D.; Haynes, C. L.; Van Duyne, R. P., Towards advanced chemical and biological nanosensors - An overview. Talanta 2005, 67, 438-448.

23. Haes, A. J.; Stuart, D. A.; Nie, S. M.; Van Duyne, R. P., Using solution-phase nanoparticles, surface-confined nanoparticle arrays and single nanoparticles as biological sensing platforms. Journal of Fluorescence 2004, 14, 355-367.

24. Szunerits, S.; Boukherroub, R., Sensing using localised surface plasmon resonance sensors. Chemical Communications 2012, 48, 8999-9010.

25. Petryayeva, E.; Krull, U. J., Localized surface plasmon resonance: Nanostructures, bioassays and biosensing-A review. Analytica Chimica Acta 2011, 706, 8-24.

26. Stewart, M. E.; Anderton, C. R.; Thompson, L. B.; Maria, J.; Gray, S. K.; Rogers, J. A.; Nuzzo, R. G., Nanostructured plasmonic sensors. Chemical Reviews 2008, 108, 494-521.

27. Haes, A. J.; Hall, W. P.; Chang, L.; Klein, W. L.; Van Duyne, R. P., A localized surface plasmon resonance biosensor: First steps toward an assay for Alzheimer's disease. Nano Letters 2004, 4, 1029-1034.

28. Haes, A. J.; Chang, L.; Klein, W. L.; Van Duyne, R. P., Detection of a biomarker for Alzheimer's disease from synthetic and clinical samples using a nanoscale optical biosensor. Journal of the American Chemical Society 2005, 127, 2264-2271.

29. Acimovic, S. S.; Ortega, M. A.; Sanz, V.; Berthelot, J.; Garcia-Cordero, J. L.; Renger, J.; Maerkl, S. J.; Kreuzer, M. P.; Quidant, R., LSPR Chip for Parallel, Rapid, and Sensitive Detection of Cancer Markers in Serum. Nano Letters 2014, 14, 2636-2641.

30. Chen, H. J.; Kou, X. S.; Yang, Z.; Ni, W. H.; Wang, J. F., Shape- and size-dependent refractive index sensitivity of gold nanoparticles. Langmuir 2008, 24, 5233-5237. 
31. Jain, P. K.; El-Sayed, M. A., Plasmonic coupling in noble metal nanostructures. Chemical Physics Letters 2010, 487, 153-164.

32. Faraday, M., The Bakerian Lecture: Experimental Relations of Gold (and Other Metals) to Light. Philos. T. Roy. Soc. 1857, 147, 145-181.

33. Mappes, T.; Jahr, N.; Csaki, A.; Vogler, N.; Popp, J.; Fritzsche, W., The Invention of Immersion Ultramicroscopy in 1912-The Birth of Nanotechnology? Angewandte ChemieInternational Edition 2012, 51, 11208-11212.

34. Dahl, J. A.; Maddux, B. L. S.; Hutchison, J. E., Toward greener nanosynthesis. Chemical Reviews 2007, 107, 2228-2269.

35. Rycenga, M.; Cobley, C. M.; Zeng, J.; Li, W. Y.; Moran, C. H.; Zhang, Q.; Qin, D.; Xia, Y. N., Controlling the Synthesis and Assembly of Silver Nanostructures for Plasmonic Applications. Chemical Reviews 2011, 111, 3669-3712.

36. Henzie, J.; Lee, J.; Lee, M. H.; Hasan, W.; Odom, T. W., Nanofabrication of Plasmonic Structures. In Annual Review of Physical Chemistry, Annual Reviews: Palo Alto, 2009; Vol. 60, pp 147-165.

37. Stewart, M. E.; Motala, M. J.; Yao, J.; Thompson, L. B.; Nuzzo, R. G., Unconventional Methods for Forming Nanopatterns. Proceedings of the Institution of Mechanical Engineers, Part N: Journal of Nanoengineering and Nanosystems 2006, 220, 81-138.

38. Pease, R. F.; Chou, S. Y., Lithography and other patterning techniques for future electronics. Proceedings of the Ieee 2008, 96, 248-270.

39. Gunnarsson, L.; Bjerneld, E. J.; Xu, H.; Petronis, S.; Kasemo, B.; Kall, M., Interparticle coupling effects in nanofabricated substrates for surface-enhanced Raman scattering. Applied Physics Letters 2001, 78, 802-804.

40. Felidj, N.; Aubard, J.; Levi, G.; Krenn, J. R.; Salerno, M.; Schider, G.; Lamprecht, B.; Leitner, A.; Aussenegg, F. R., Controlling the optical response of regular arrays of gold particles for surface-enhanced Raman scattering. Physical Review B 2002, 65, 9.

41. Abu Hatab, N. A.; Oran, J. M.; Sepaniak, M. J., Surface-enhanced Raman spectroscopy substrates created via electron beam lithography and nanotransfer printing. Acs Nano 2008, 2, 377-385.

42. Shankar, S. S.; Rizzello, L.; Cingolani, R.; Rinaldi, R.; Pompa, P. P., Micro/Nanoscale Patterning of Nanostructured Metal Substrates for Plasmonic Applications. Acs Nano 2009, 3, 893-900.

43. Corrigan, T. D.; Guo, S.; Phaneuf, R. J.; Szmacinski, H., Enhanced fluorescence from periodic arrays of silver nanoparticles. Journal of Fluorescence 2005, 15, 777-784.

44. Acimovic, S. S.; Kreuzer, M. P.; Gonzalez, M. U.; Quidant, R., Plasmon Near-Field Coupling in Metal Dimers as a Step toward Single-Molecule Sensing. Acs Nano 2009, 3, 1231-1237.

45. Piliarik, M.; Kvasnicka, P.; Galler, N.; Krenn, J. R.; Homola, J., Local refractive index sensitivity of plasmonic nanoparticles. Optics Express 2011, 19, 9213-9220. 
46. Haynes, C. L.; Van Duyne, R. P., Nanosphere lithography: A versatile nanofabrication tool for studies of size-dependent nanoparticle optics. Journal of Physical Chemistry B 2001, 105, 5599-5611.

47. Hulteen, J. C.; Vanduyne, R. P., Nanosphere lithography - a materials general fabrication process for periodic particle array surfaces. Journal of Vacuum Science \& Technology aVacuum Surfaces and Films 1995, 13, 1553-1558.

48. Ormonde, A. D.; Hicks, E. C. M.; Castillo, J.; Van Duyne, R. P., Nanosphere lithography: Fabrication of large-area Ag nanoparticle arrays by convective self-assembly and their characterization by scanning UV-visible extinction spectroscopy. Langmuir 2004, 20, 69276931.

49. Whatmore, R. W., Nanotechnology: big prospects for small engineering Ingenia 2001, 28-34.

50. Zhao, P. X.; Li, N.; Astruc, D., State of the art in gold nanoparticle synthesis. Coordination Chemistry Reviews 2013, 257, 638-665.

51. Sun, Y. G.; Xia, Y. N., Shape-controlled synthesis of gold and silver nanoparticles. Science 2002, 298, 2176-2179.

52. Turkevich, J.; Stevenson, P. C.; Hillier, J., A study of the nucleation and growth processes in the synthesis of colloidal gold. Discussions of the Faraday Society 1951, 11, 55-75.

53. Frens, G., Controlled Nucleation for the Regulation of the Particle Size in Monodisperse Gold Suspensions. Nature Physical Science 1973, 241, 20-22.

54. Ji, X. H.; Song, X. N.; Li, J.; Bai, Y. B.; Yang, W. S.; Peng, X. G., Size control of gold nanocrystals in citrate reduction: The third role of citrate. Journal of the American Chemical Society 2007, 129, 13939-13948.

55. Hu, M.; Chen, J. Y.; Li, Z. Y.; Au, L.; Hartland, G. V.; Li, X. D.; Marquez, M.; Xia, Y. N., Gold nanostructures: engineering their plasmonic properties for biomedical applications. Chemical Society Reviews 2006, 35, 1084-1094.

56. Jana, N. R.; Gearheart, L.; Murphy, C. J., Wet chemical synthesis of high aspect ratio cylindrical gold nanorods. Journal of Physical Chemistry B 2001, 105, 4065-4067.

57. Jana, N. R.; Gearheart, L.; Murphy, C. J., Seed-mediated growth approach for shapecontrolled synthesis of spheroidal and rod-like gold nanoparticles using a surfactant template. Advanced Materials 2001, 13, 1389-1393.

58. Nikoobakht, B.; El-Sayed, M. A., Preparation and growth mechanism of gold nanorods (NRs) using seed-mediated growth method. Chemistry of Materials 2003, 15, 1957-1962.

59. Zhang, J. A.; Langille, M. R.; Personick, M. L.; Zhang, K.; Li, S. Y.; Mirkin, C. A., Concave Cubic Gold Nanocrystals with High-Index Facets. Journal of the American Chemical Society 2010, 132, 14012-14014.

60. Nehl, C. L.; Liao, H. W.; Hafner, J. H., Optical properties of star-shaped gold nanoparticles. Nano Letters 2006, 6, 683-688. 
61. Ha, T. H.; Koo, H. J.; Chung, B. H., Shape-controlled syntheses of gold nanoprisms and nanorods influenced by specific adsorption of halide ions. Journal of Physical Chemistry C 2007, 111, 1123-1130.

62. Niidome, T.; Yamagata, M.; Okamoto, Y.; Akiyama, Y.; Takahashi, H.; Kawano, T.; Katayama, Y.; Niidome, Y., PEG-modified gold nanorods with a stealth character for in vivo applications. Journal of Controlled Release 2006, 114, 343-347.

63. Takahashi, H.; Niidome, Y.; Niidome, T.; Kaneko, K.; Kawasaki, H.; Yamada, S., Modification of gold nanorods using phospatidylcholine to reduce cytotoxicity. Langmuir 2006, 22, 2-5.

64. Hauck, T. S.; Ghazani, A. A.; Chan, W. C. W., Assessing the effect of surface chemistry on gold nanorod uptake, toxicity, and gene expression in mammalian cells. Small 2008, 4, 153159.

65. Gole, A.; Murphy, C. J., Polyelectrolyte-coated gold nanorods: Synthesis, characterization and immobilization. Chemistry of Materials 2005, 17, 1325-1330.

66. Hrelescu, C.; Sau, T. K.; Rogach, A. L.; Jackel, F.; Laurent, G.; Douillard, L.; Charra, F., Selective Excitation of Individual Plasmonic Hotspots at the Tips of Single Gold Nanostars. Nano Letters 2011, 11, 402-407.

67. Guedje, F. K.; Giloan, M.; Potara, M.; Hounkonnou, M. N.; Astilean, S., Optical properties of single silver triangular nanoprism. Physica Scripta 2012, 86, 6.

68. Jin, R. C.; Cao, Y. W.; Mirkin, C. A.; Kelly, K. L.; Schatz, G. C.; Zheng, J. G., Photoinduced conversion of silver nanospheres to nanoprisms. Science 2001, 294, 1901-1903.

69. Millstone, J. E.; Hurst, S. J.; Metraux, G. S.; Cutler, J. I.; Mirkin, C. A., Colloidal Gold and Silver Triangular Nanoprisms. Small 2009, 5, 646-664.

70. Shahjamali, M. M.; Bosman, M.; Cao, S. W.; Huang, X.; Saadat, S.; Martinsson, E.; Aili, D.; Tay, Y. Y.; Liedberg, B.; Loo, S. C. J.; Zhang, H.; Boey, F.; Xue, C., Gold Coating of Silver Nanoprisms. Advanced Functional Materials 2012, 22, 849-854.

71. Enoch, S.; Bonod, N., Plasmonics: From Basics to Advanced Topics. Springer: Heidelberg, 2012; Vol. 167.

72. Raether, H., Surface Plasmons on Smooth and Rough Surfaces and on Gratings. SpringerVerlag: 1988.

73. Kretschmann, E.; Raether, H., Radiative decay of non radiative surface plasmons excited by light. Zeitschrift für Naturforschung A 1968, 23a, 2135-2136.

74. Otto, A., Excitation of Nonradiative Surface Plasma Waves in Silver by the Method of Frustrated Total Reflection. Zeitschrift für Physik. A, Hadrons and Nuclei 1968, 216, 398-410.

75. Teng, Y. Y.; Stern, E. A., Plasma Radiation from Metal Grating Surfaces. Physical Review Letters 1967, 19, 511-514.

76. Mie, G., Beiträge zur Optik trüber Medien, speziell kolloidaler Metallösungen. Annalen der Physik, 1908; Vol. 25, pp 377-445. 
77. $\quad$ Dmitriev, A., Nanoplasmonic Sensors. Springer: New York, 2012.

78. Sonnichsen, C.; Franzl, T.; Wilk, T.; von Plessen, G.; Feldmann, J.; Wilson, O.; Mulvaney, P., Drastic reduction of plasmon damping in gold nanorods. Physical Review Letters 2002, 88, 4.

79. Meier, M.; Wokaun, A., Enhanced fields on large metal particles: dynamic depolarization Optics Letters 1983, 8, 581-583.

80. Zhang, J. Z.; Noguez, C., Plasmonic Optical Properties and Applications of Metal Nanostructures. Plasmonics 2008, 3, 127-150.

81. Shuford, K. L.; Ratner, M. A.; Schatz, G. C., Multipolar excitation in triangular nanoprisms. Journal of Chemical Physics 2005, 123, 9.

82. Wei, H.; Reyes-Coronado, A.; Nordlander, P.; Aizpurua, J.; Xu, H. X., Multipolar Plasmon Resonances in Individual Ag Nanorice. Acs Nano 2010, 4, 2649-2654.

83. Krenn, J. R.; Schider, G.; Rechberger, W.; Lamprecht, B.; Leitner, A.; Aussenegg, F. R.; Weeber, J. C., Design of multipolar plasmon excitations in silver nanoparticles. Applied Physics Letters 2000, 77, 3379-3381.

84. Schatz, G. C., Electrodynamics of nonspherical noble metal nanoparticles and nanoparticle aggregates. Journal of Molecular Structure-Theochem 2001, 573, 73-80.

85. Jain, P. K.; Lee, K. S.; El-Sayed, I. H.; El-Sayed, M. A., Calculated absorption and scattering properties of gold nanoparticles of different size, shape, and composition: Applications in biological imaging and biomedicine. Journal of Physical Chemistry B 2006, 110, 7238-7248.

86. Kelly, K. L.; Coronado, E.; Zhao, L. L.; Schatz, G. C., The optical properties of metal nanoparticles: The influence of size, shape, and dielectric environment. Journal of Physical Chemistry B 2003, 107, 668-677.

87. Krug, J. T.; Sanchez, E. J.; Xie, X. S., Design of near-field optical probes with optimal field enhancement by finite difference time domain electromagnetic simulation. Journal of Chemical Physics 2002, 116, 10895-10901.

88. Oubre, C.; Nordlander, P., Optical properties of metallodielectric nanostructures calculated using the finite difference time domain method. Journal of Physical Chemistry B 2004, 108, 17740-17747.

89. Chang, S. H.; Gray, S. K.; Schatz, G. C., Surface plasmon generation and light transmission by isolated nanoholes and arrays of nanoholes in thin metal films. Optics Express 2005, 13, 3150-3165.

90. Prodan, E.; Radloff, C.; Halas, N. J.; Nordlander, P., A hybridization model for the plasmon response of complex nanostructures. Science 2003, 302, 419-422.

91. Ghosh, S. K.; Pal, T., Interparticle coupling effect on the surface plasmon resonance of gold nanoparticles: From theory to applications. Chemical Reviews 2007, 107, 4797-4862.

92. Esteban, R.; Borisov, A. G.; Nordlander, P.; Aizpurua, J., Bridging quantum and classical plasmonics with a quantum-corrected model. Nature Communications 2012, 3, 9. 
93. Camden, J. P.; Dieringer, J. A.; Wang, Y. M.; Masiello, D. J.; Marks, L. D.; Schatz, G. C.; Van Duyne, R. P., Probing the structure of single-molecule surface-enhanced Raman scattering hot spots. Journal of the American Chemical Society 2008, 130, 12616-12617.

94. Li, W. Y.; Camargo, P. H. C.; Lu, X. M.; Xia, Y. N., Dimers of Silver Nanospheres: Facile Synthesis and Their Use as Hot Spots for Surface-Enhanced Raman Scattering. Nano Letters 2009, 9, 485-490.

95. Mirkin, C. A.; Letsinger, R. L.; Mucic, R. C.; Storhoff, J. J., A DNA-based method for rationally assembling nanoparticles into macroscopic materials. Nature 1996, 382, 607-609.

96. Aili, D.; Selegard, R.; Baltzer, L.; Enander, K.; Liedberg, B., Colorimetric Protein Sensing by Controlled Assembly of Gold Nanoparticles Functionalized with Synthetic Receptors. Small 2009, 5, 2445-2452.

97. Chen, P.; Selegard, R.; Aili, D.; Liedberg, B., Peptide functionalized gold nanoparticles for colorimetric detection of matrilysin (MMP-7) activity. Nanoscale 2013, 5, 8973-8976.

98. Kumar, C., Biofunctionalization of Nanomaterials. Wiley-VCH Verlag: Weinheim, 2005.

99. Nuzzo, R. G.; Allara, D. L., Adsorption of bifunctional organic disulfides on gold surfaces. Journal of Chemical Society 1983, 5, 4481-4483.

100. Pastoriza-Santos, I.; Perez-Juste, J.; Liz-Marzan, L. M., Silica-coating and hydrophobation of CTAB-stabilized gold nanorods. Chemistry of Materials 2006, 18, 2465-2467.

101. Xue, C.; Chen, X.; Hurst, S. J.; Mirkin, C. A., Self-assembled monolayer mediated silica coating of silver triangular nanoprisms. Advanced Materials 2007, 19, 4071-4074.

102. Shipway, A. N.; Katz, E.; Willner, I., Nanoparticle arrays on surfaces for electronic, optical, and sensor applications. Chemphyschem 2000, 1, 18-52.

103. Hahn, M. A.; Singh, A. K.; Sharma, P.; Brown, S. C.; Moudgil, B. M., Nanoparticles as contrast agents for in-vivo bioimaging: current status and future perspectives. Analytical and Bioanalytical Chemistry 2011, 399, 3-27.

104. Schmitt, J.; Machtle, P.; Eck, D.; Mohwald, H.; Helm, C. A., Preparation and optical properties of colloidal gold monolayers. Langmuir 1999, 15, 3256-3266.

105. Grabar, K. C.; Smith, P. C.; Musick, M. D.; Davis, J. A.; Walter, D. G.; Jackson, M. A.; Guthrie, A. P.; Natan, M. J., Kinetic control of interparticle spacing in Au colloid-based surfaces: Rational nanometer-scale architecture. Journal of the American Chemical Society 1996, 118, 1148-1153.

106. Lundgren, A.; Hulander, M.; Brorsson, J.; Hermansson, M.; Elwing, H.; Andersson, O.; Liedberg, B.; Berglin, M., Gold-Nanoparticle-Assisted Self-Assembly of Chemical Gradients with Tunable Sub-50 nm Molecular Domains. Particle \& Particle Systems Characterization 2014, 31, 209-218.

107. Silberzan, P.; Leger, L.; Ausserre, D.; Benattar, J. J., Silanation of silica surfaces - a new method of constructing pure or mixed monolayers. Langmuir 1991, 7, 1647-1651.

108. Decher, G., Fuzzy nanoassemblies: Toward layered polymeric multicomposites. Science 1997, 277, 1232-1237. 
109. Decher, G.; Hong, J. D.; Schmitt, J., Buildup of ultrathin multilayer films by a self-assembly process. III Consecutively alternating adsorption of anionic and cationic polyelectrolytes on charged surfaces. Thin Solid Films 1992, 210, 831-835.

110. Smith, R. N.; McCormick, M.; Barrett, C. J.; Reven, L.; Spiess, H. W., NMR studies of PAH/PSS polyelectrolyte multilayers adsorbed onto silica. Macromolecules 2004, 37, 48304838.

111. Feldoto, Z.; Varga, I.; Blomberg, E., Influence of Salt and Rinsing Protocol on the Structure of PAH/PSS Polyelectrolyte Multilayers. Langmuir 2010, 26, 17048-17057.

112. Losche, M.; Schmitt, J.; Decher, G.; Bouwman, W. G.; Kjaer, K., Detailed structure of molecularly thin polyelectrolyte multilayer films on solid substrates as revealed by neutron reflectometry. Macromolecules 1998, 31, 8893-8906.

113. Wong, J. E.; Rehfeldt, F.; Hanni, P.; Tanaka, M.; Klitzing, R. V., Swelling behavior of polyelectrolyte multilayers in saturated water vapor. Macromolecules 2004, 37, 7285-7289.

114. Taton, T. A.; Mucic, R. C.; Mirkin, C. A.; Letsinger, R. L., The DNA-mediated formation of supramolecular mono- and multilayered nanoparticle structures. Journal of the American Chemical Society 2000, 122, 6305-6306.

115. Aili, D.; Han, H. S.; Enander, K.; Baltzer, L.; Liedberg, B. In Controlled assembly of gold nanoparticles using de novo designed polypeptide scaffolds, Conference on MEMS/MOEMS Components and Their Applications V, San Jose, CA, Jan 21-22; Spie-Int Soc Optical Engineering: San Jose, CA, 2008; pp 88506-88506.

116. Schmitt, J.; Decher, G.; Dressick, W. J.; Brandow, S. L.; Geer, R. E.; Shashidhar, R.; Calvert, J. M., Metal nanoparticle/polymer superlattice films: Fabrication and control of layer structure. Advanced Materials 1997, 9, 61-65.

117. Jiang, C. Y.; Markutsya, S.; Tsukruk, V. V., Collective and individual plasmon resonances in nanoparticle films obtained by spin-assisted layer-by-layer assembly. Langmuir 2004, 20, 882890.

118. Thevenot, D. R.; Toth, K.; Durst, R. A.; Wilson, G. S., Electrochemical biosensors: recommended definitions and classification. Biosensors \& Bioelectronics 2001, 16, 121-131.

119. Dahlin, A. B., Plasmonic Biosensors: An Integrated View of Refractometric Detection. IOS Press: 2012.

120. Clark Jr, L. C.; Lyons, C., Electrode Systems for Continuous Monitoring in Cardiovascular Surgery. Annals New York Academy of Sciences 1962, 102, 29-45.

121. Wang, J., Glucose biosensors: 40 years of advances and challenges. Electroanalysis 2001, 13, 983-988.

122. Filippini, D.; Svensson, S. P. S.; Lundstrom, I., Computer screen as a programmable light source for visible absorption characterization of (bio)chemical assays. Chemical Communications 2003, 240-241.

123. Preechaburana, P.; Gonzalez, M. C.; Suska, A.; Filippini, D., Surface Plasmon Resonance Chemical Sensing on Cell Phones. Angewandte Chemie-International Edition 2012, 51, 11585-11588. 
124. Harder, P.; Grunze, M.; Dahint, R.; Whitesides, G. M.; Laibinis, P. E., Molecular conformation in oligo(ethylene glycol)-terminated self-assembled monolayers on gold and silver surfaces determines their ability to resist protein adsorption. Journal of Physical Chemistry B 1998, 102, 426-436.

125. Love, J. C.; Estroff, L. A.; Kriebel, J. K.; Nuzzo, R. G.; Whitesides, G. M., Self-assembled monolayers of thiolates on metals as a form of nanotechnology. Chemical Reviews 2005, 105, 1103-1169.

126. Wang, Z. H.; Jin, G., Silicon surface modification with a mixed silanes layer to immobilize proteins for biosensor with imaging ellipsometry. Colloids and Surfaces B-Biointerfaces 2004, 34, 173-177.

127. Lofas, S.; Johnsson, B., A novel hydrogel matrix on gold surfaces in surface-plasmon resonance sensors for fast and efficient covalent immobilization of ligands. Journal of the Chemical Society-Chemical Communications 1990, 1526-1528.

128. Larsson, A.; Ekblad, T.; Andersson, O.; Liedberg, B., Photografted poly(ethylene glycol) matrix for affinity interaction studies. Biomacromolecules 2007, 8, 287-295.

129. Zhang, S. G., Hydrogels - Wet or let die. Nature Materials 2004, 3, 7-8.

130. Liedberg, B.; Nylander, C.; Lundstrom, I., Surface-plasmon resonance for gas-detection and biosensing. Sensors and Actuators 1983, 4, 299-304.

131. Homola, J., Surface plasmon resonance sensors for detection of chemical and biological species. Chemical Reviews 2008, 108, 462-493.

132. Englebienne, P., Use of colloidal gold surface plasmon resonance peak shift to infer affinity constants from the interactions between protein antigens and antibodies specific for single or multiple epitopes. Analyst 1998, 123, 1599-1603.

133. Ferreira, J.; Santos, M. J. L.; Rahman, M. M.; Brolo, A. G.; Gordon, R.; Sinton, D.; Girotto, E. M., Attomolar Protein Detection Using in-Hole Surface Plasmon Resonance. Journal of the American Chemical Society 2009, 131, 436-437.

134. Nath, N.; Chilkoti, A., Label-free biosensing by surface plasmon resonance of nanoparticles on glass: Optimization of nanoparticle size. Analytical Chemistry 2004, 76, 5370-5378.

135. Otte, M. A.; Estevez, M. C.; Carrascosa, L. G.; Gonzalez-Guerrero, A. B.; Lechuga, L. M.; Sepulveda, B., Improved Biosensing Capability with Novel Suspended Nanodisks. Journal of Physical Chemistry C 2011, 115, 5344-5351.

136. Olofsson, L.; Rindzevicius, T.; Pfeiffer, I.; Kall, M.; Hook, F., Surface-based goldnanoparticle sensor for specific and quantitative DNA hybridization detection. Langmuir 2003, 19, 10414-10419.

137. Dahlin, A. B.; Tegenfeldt, J. O.; Hook, F., Improving the instrumental resolution of sensors based on localized surface plasmon resonance. Analytical Chemistry 2006, 78, 4416-4423.

138. Satija, J.; Punjabi, N. S.; Sai, V. V. R.; Mukherji, S., Optimal Design for U-bent Fiber-optic LSPR Sensor Probes. Plasmonics 2014, 9, 251-260. 
139. Chen, P.; Liedberg, B., Curvature of the Localized Surface Plasmon Resonance Peak. Analytical Chemistry 2014, 86, 7399-7405.

140. McFarland, A. D.; Van Duyne, R. P., Single silver nanoparticles as real-time optical sensors with zeptomole sensitivity. Nano Letters 2003, 3, 1057-1062.

141. Sherry, L. J.; Jin, R. C.; Mirkin, C. A.; Schatz, G. C.; Van Duyne, R. P., Localized surface plasmon resonance spectroscopy of single silver triangular nanoprisms. Nano Letters 2006, 6, 2060-2065.

142. Chan, G. H.; Zhao, J.; Schatz, G. C.; Van Duyne, R. P., Localized surface plasmon resonance spectroscopy of triangular aluminum nanoparticles. Journal of Physical Chemistry C 2008, 112, 13958-13963.

143. Zoric, I.; Zach, M.; Kasemo, B.; Langhammer, C., Gold, Platinum, and Aluminum Nanodisk Plasmons: Material Independence, Subradiance, and Damping Mechanisms. Acs Nano 2011, 5, 2535-2546.

144. Lu, X. M.; Rycenga, M.; Skrabalak, S. E.; Wiley, B.; Xia, Y. N., Chemical Synthesis of Novel Plasmonic Nanoparticles. In Annual Review of Physical Chemistry, Annual Reviews: Palo Alto, 2009; Vol. 60, pp 167-192.

145. Faucheaux, J. A.; Stanton, A. L. D.; Jain, P. K., Plasmon Resonances of Semiconductor Nanocrystals: Physical Principles and New Opportunities. Journal of Physical Chemistry Letters 2014, 5, 976-985.

146. Miller, M. M.; Lazarides, A. A., Sensitivity of metal nanoparticle surface plasmon resonance to the dielectric environment. Journal of Physical Chemistry B 2005, 109, 21556-21565.

147. Charles, D. E.; Aherne, D.; Gara, M.; Ledwith, D. M.; Gun'ko, Y. K.; Kelly, J. M.; Blau, W. J.; Brennan-Fournet, M. E., Versatile Solution Phase Triangular Silver Nanoplates for Highly Sensitive Plasmon Resonance Sensing. Acs Nano 2010, 4, 55-64.

148. Sannomiya, T.; Sahoo, P. K.; Mahcicek, D. I.; Solak, H. H.; Hafner, C.; Grieshaber, D.; Voros, J., Biosensing by Densely Packed and Optically Coupled Plasmonic Particle Arrays. Small 2009, 5, 1889-1896.

149. Tesler, A. B.; Chuntonov, L.; Karakouz, T.; Bendikov, T. A.; Haran, G.; Vaskevich, A.; Rubinstein, I., Tunable Localized Plasmon Transducers Prepared by Thermal Dewetting of Percolated Evaporated Gold Films. Journal of Physical Chemistry C 2011, 115, 24642-24652.

150. Jakab, A.; Rosman, C.; Khalavka, Y.; Becker, J.; Trugler, A.; Hohenester, U.; Sonnichsen, C., Highly Sensitive Plasmonic Silver Nanorods. Acs Nano 2011, 5, 6880-6885.

151. Otte, M. A.; Sepulveda, B.; Ni, W. H.; Juste, J. P.; Liz-Marzan, L. M.; Lechuga, L. M., Identification of the Optimal Spectral Region for Plasmonic and Nanoplasmonic Sensing. Acs Nano 2010, 4, 349-357.

152. Chen, H. J.; Ming, T.; Zhang, S. R.; Jin, Z.; Yang, B. C.; Wang, J. F., Effect of the Dielectric Properties of Substrates on the Scattering Patterns of Gold Nanorods. Acs Nano 2011, 5, 48654877. 
153. Valamanesh, M.; Borensztein, Y.; Langlois, C.; Lacaze, E., Substrate Effect on the Plasmon Resonance of Supported Flat Silver Nanoparticles. Journal of Physical Chemistry C 2011, 115, 2914-2922.

154. Brian, B.; Sepulveda, B.; Alaverdyan, Y.; Lechuga, L. M.; Kall, M., Sensitivity enhancement of nanoplasmonic sensors in low refractive index substrates. Optics Express 2009, 17, 20152023.

155. Haes, A. J.; Zou, S. L.; Schatz, G. C.; Van Duyne, R. P., A nanoscale optical biosensor: The long range distance dependence of the localized surface plasmon resonance of noble metal nanoparticles. Journal of Physical Chemistry B 2004, 108, 109-116.

156. Brolo, A. G., Plasmonics for future biosensors. Nature Photonics 2012, 6, 709-713.

157. Rang, M.; Jones, A. C.; Zhou, F.; Li, Z. Y.; Wiley, B. J.; Xia, Y. N.; Raschke, M. B., Optical Near-Field Mapping of Plasmonic Nanoprisms. Nano Letters 2008, 8, 3357-3363.

158. Feuz, L.; Jonsson, P.; Jonsson, M. P.; Hook, F., Improving the Limit of Detection of Nanoscale Sensors by Directed Binding to High-Sensitivity Areas. Acs Nano 2010, 4, $2167-$ 2177.

159. Zhang, N.; Liu, Y. J.; Yang, J.; Su, X. D.; Deng, J.; Chum, C. C.; Hong, M. H.; Teng, J. H., High sensitivity molecule detection by plasmonic nanoantennas with selective binding at electromagnetic hotspots. Nanoscale 2014, 6, 1416-1422.

160. Okamoto, T.; Yamaguchi, I.; Kobayashi, T., Local plasmon sensor with gold colloid monolayers deposited upon glass substrates. Optics Letters 2000, 25, 372-374.

161. Kedem, O.; Tesler, A. B.; Vaskevich, A.; Rubinstein, I., Sensitivity and Optimization of Localized Surface Plasmon Resonance Transducers. Acs Nano 2011, 5, 748-760.

162. Tian, L. M.; Chen, E.; Gandra, N.; Abbas, A.; Singamaneni, S., Gold Nanorods as Plasmonic Nanotransducers: Distance-Dependent Refractive Index Sensitivity. Langmuir 2012, 28, 17435-17442.

163. Evanoff, D. D.; White, R. L.; Chumanov, G., Measuring the distance dependence of the local electromagnetic field from silver nanoparticles. Journal of Physical Chemistry B 2004, 108, 1522-1524.

164. Bukasov, R.; Ali, T. A.; Nordlander, P.; Shumaker-Parry, J. S., Probing the Plasmonic NearField of Gold Nanocrescent Antennas. Acs Nano 2010, 4, 6639-6650.

165. Malinsky, M. D.; Kelly, K. L.; Schatz, G. C.; Van Duyne, R. P., Chain length dependence and sensing capabilities of the localized surface plasmon resonance of silver nanoparticles chemically modified with alkanethiol self-assembled monolayers. Journal of the American Chemical Society 2001, 123, 1471-1482.

166. Svedendahl, M.; Chen, S.; Dmitriev, A.; Kall, M., Refractometric Sensing Using Propagating versus Localized Surface Plasmons: A Direct Comparison. Nano Letters 2009, 9, 4428-4433.

167. Kabashin, A. V.; Evans, P.; Pastkovsky, S.; Hendren, W.; Wurtz, G. A.; Atkinson, R.; Pollard, R.; Podolskiy, V. A.; Zayats, A. V., Plasmonic nanorod metamaterials for biosensing. Nature Materials 2009, 8, 867-871. 
168. Hall, W. P.; Anker, J. N.; Lin, Y.; Modica, J.; Mrksich, M.; Van Duyne, R. P., A calciummodulated plasmonic switch. Journal of the American Chemical Society 2008, 130, 58365837. 


\section{Publications}

The articles associated with this thesis have been removed for copyright reasons. For more details about these see:

http://urn.kb.se/resolve?urn=urn:nbn:se:liu:diva-111841 